\title{
The Highly Unusual Outgassing of Comet 103P/Hartley 2 from Narrowband Photometry and Imaging of the Coma
}

\author{
Matthew M. Knight ${ }^{1,2,3}$ and David G. Schleicher ${ }^{2}$
}

\begin{abstract}
We report on photometry and imaging of Comet 103P/Hartley 2 obtained at Lowell Observatory from 1991 through 2011. We acquired photoelectric photometry on two nights in 1991, four nights in 1997/98, and 13 nights in 2010/11. We observed a strong secular decrease in water and all other observed species production in 2010/11 from the 1991 and 1997/98 levels. We see evidence for a strong asymmetry with respect to perihelion in the production rates of our usual bandpasses, with peak production occurring $\sim 10$ days postperihelion and production rates considerably higher post-perihelion. The composition was "typical," in agreement with the findings of other investigators. We obtained imaging on 39 nights from 2010 July until 2011 January. We find that, after accounting for their varying parentage and lifetimes, the $\mathrm{C}_{2}$ and $\mathrm{C}_{3}$ coma morphology resemble the CN morphology we reported previously. These species exhibited an hourglass shape in October and November, and the morphology changed with rotation and evolved over time. The $\mathrm{OH}$ and $\mathrm{NH}$ coma morphology showed hints of an hourglass shape near the nucleus, but was also enhanced in the anti-sunward hemisphere. This tailward brightness enhancement did not vary significantly with rotation and evolved with the viewing geometry. We conclude that all five gas species likely originate from the same source regions on the nucleus, but that $\mathrm{OH}$ and $\mathrm{NH}$ were derived from small grains of water and ammonia ice that survived long enough to be affected by radiation pressure and driven in the anti-sunward direction. We detected the faint, sunward facing dust jet reported by other authors, and did not detect a corresponding gas feature. This jet varied little during a night but exhibited some variations from night to night, suggesting it is located near the total angular momentum vector. Overall, our imaging results support the conclusions of other authors that Hartley 2's "hyperactivity" is caused by icy particles of various sizes that are lifted off the surface and break up in the coma to greatly increase the effective active surface area.

\footnotetext{
${ }^{1}$ Contacting author: knight@lowell.edu.

${ }^{2}$ Lowell Observatory, 1400 W. Mars Hill Rd, Flagstaff, AZ 86001, U.S.A.

${ }^{3}$ The Johns Hopkins University Applied Physics Laboratory, 11100 Johns Hopkins Road, Laurel, Maryland 20723
} 
Keywords: Comets; Comets, coma; Comets, composition

\section{INTRODUCTION}

Jupiter-family comet 103P/Hartley 2 became one of the best-studied comets of all time when, in late-2010, it simultaneously passed very close to the Earth and was the subject of a flyby by the EPOXI spacecraft (e.g., Meech et al. 2011). First results have already been published for EPOXI spacecraft data (A'Hearn et al. 2011), radar observations of the nucleus (Harmon et al. 2011), composition of the coma Combi et al. 2011a; Dello Russo et al. 2011; Drahus et al. 2011; Mumma et al.|2011; Weaver et al. 2011; Hartogh et al. 2011), and morphology of the coma (Knight and Schleicher 2011; Samarasinha et al. 2011; Lara et al. 2011; Waniak et al. 2012). These and earlier results will undoubtedly be discussed in more detail elsewhere in this Special Issue; in the interest of brevity, we restrict our discusion to those results having bearing on our current work.

We obtained both photometry and imaging of Hartley 2, and discuss the observations and reductions in Section 2. In Section 3 we discuss our photoelectric photometer measurements acquired during the 1991, 1997/98, and 2010/11 apparitions. Due to unfavorable viewing geometry, we did not observe Hartley 2 on its two other known apparition, 1986 (when it was discovered) or 2004. Previous investigators (Weaver et al. 1994; A'Hearn et al. 1995) found a typical composition and a low dust-to-gas ratio. Assuming that Hartley 2 had a comparable active fraction to other comets $(\sim 10 \%)$, it was inferred to have a rather large nucleus (of order a few $\mathrm{km}$ ). The EPOXI flyby conclusively showed that Hartley 2 has a small nucleus (effective radius of $0.57 \pm 0.02 \mathrm{~km}$; A'Hearn et al. 2011), confirming the conclusions of remote studies of the nucleus (Groussin et al. 2004; Lisse et al. 2009). EPOXI also revealed that the active fraction of the nucleus is relatively low and thus its "hyperactivity" necessitates an extended source of volatiles. Numerous new water production measurements have recently been published in support of EPOXI and are compared with our measurements.

We also follow up our previous investigation of the CN coma morphology in the 2010/2011 apparition. We discovered the existence of time-varying CN coma structures and used the repetition of these structures to measure a rotation period (Knight et al. 2010). In Knight and Schleicher (2011), henceforth "Paper 1", we interpreted the morphology as likely being due to two "jets" whose appearance varied as the nucleus rotated, and evolved during the apparition. We used repetitions in the morphology to measure rotation periods monthly from 2010 August through 2010 November and determined that the rotation period was increasing during this time from $\sim 16.7 \mathrm{hr}$ in August to $\sim 18.7 \mathrm{hr}$ in November. We noted that the morphology did not exactly repeat from cycle to cycle, but repeated better after 
$3,6,9 \ldots$ cycles and inferred that it was in non-principal axis rotation with a component period roughly three times as long as the measured rotation period. Similar coma morphology and rotation periods were reported by Samarasinha et al. (2011), whose images covered a comparable range of dates (2010 September 1 through December 15). Lara et al. (2011) acquired snapshot imaging near perigee, reporting similar CN morphology and showing that the $\mathrm{C}_{3}$ coma morphology also resembled the $\mathrm{CN}$ coma morphology. Lara et al. also reported the existence of a much smaller-scale dust jet in the sunward direction. A similar feature is visible in movies made from EPOXI HRI images posted on the EPOXI websit€1, Waniak et al. (2012) obtained $\mathrm{CN}$ and $\mathrm{C}_{3}$ images near the time of the EPOXI flyby, finding a similar periodicity as we reported and noting the agreement of the $\mathrm{CN}$ and $\mathrm{C}_{3}$ morphology. In Section 4 we intercompare the coma morphology exhibited by various gas species (CN, $\mathrm{C}_{3}, \mathrm{C}_{2}, \mathrm{OH}$, and $\mathrm{NH}$ ) in 2010 October and November, and discuss the dust morphology, in particular looking for evidence of the dust jet.

Finally, in Section 5 we discuss our results and how they fit into the larger body of knowledge about Hartley 2 acquired by EPOXI and numerous remote observers. We summarize our findings in Section 6 and briefly discuss their application to future modeling efforts.

\section{OBSERVATIONS AND REDUCTIONS}

\subsection{Instrumentation}

The goals of our studies of Hartley 2 have varied, with composition and production rate studies the emphasis of the 1991 and 1997/98 apparitions and multiwavelength imaging the emphasis in 2010/11 (with production rate and compositional monitoring a secondary emphasis). Thus, our techniques and instrumentation have varied. The production rate and composition studies continue to be made with a traditional photoelectric photometer. This provides superior signal-to-noise for bulk coma measurements as compared to a CCD and also ensures continuity with earlier data sets. The imaging necessarily requires use of a CCD. All photometer observations were obtained with the Hall 42-in (1.1-m) telescope at Lowell Observatory except for one night in 1997 which was made using Lowell's 0.9-m telescope at Perth Observatory. Imaging observations were acquired with either the Hall 42-in or the 31-in (0.8-m) telescopes at Lowell Observatory. In total, we acquired two nights of photometry in 1991, four nights of photometry in 1997/98, 13 nights of photometry in

\footnotetext{
${ }^{1}$ http://epoxi.umd.edu/
} 
2010/11, and 39 nights of imaging in 2010/11. The observing circumstances for all nights of imaging and photometry are given in Tables 1 and 2, respectively.

The photometers used had EMI 6256 photomultiplier tubes and pulse counting systems except for the sole night (1997 December 1) at Perth Observatory where a DC amplifier was employed. The Hale-Bopp (HB) narrowband filter set (Farnham et al. 2000) was utilized in 1997 and later, superceding the International Halley Watch (IHW) set (A'Hearn 1991) used in 1991. HB filters were also used for the CCD observations, along with a broadband Cousins $\mathrm{R}$ filter for increased signal-to-noise for dust measurements. The narrowband comet filters used for both our photometry and imaging isolate emission from daughter gas species $\left(\mathrm{OH}, \mathrm{NH}, \mathrm{CN}, \mathrm{C}_{3}, \mathrm{C}_{2}\right)$ or reflected solar continuum from dust (continuum filters in the UV (UC), blue (BC), green ( $\mathrm{GC})$, and red $(\mathrm{RC})$ ). The comet filters are regularly used to study dust and coma in comets (e.g., Woodney et al. 2002; Schleicher et al. 2006, Farnham et al. 2007; Lederer et al. 2009; Waniak et al. 2009). Using the method described in A'Hearn et al. (1995) and Farnham et al. (2000), we "decontaminate" the data by removing the underlying signal due to reflected solar continuum (and in the case of CN and NH, contamination from $\mathrm{C}_{3}$ as well) from gas bandpasses to yield pure gas and pure dust data.

The 1.1-m images were obtained using an e2v CCD231-84 chip with $4 \mathrm{k} \times 4 \mathrm{k}$ pixels which was binned $2 \times 2$ at the telescope, resulting in a pixel scale of 0.74 arcsec. The $0.8-\mathrm{m}$ images were obtained with an $\mathrm{e} 2 \mathrm{v}$ CCD $42-40$ chip with $2 \mathrm{k} \times 2 \mathrm{k}$ pixels and a pixel scale of 0.46 arcsec. At times images were rebinned by an additional factor of 2 during processing to improve the effective signal-to-noise; the final pixel scales of these images were 1.48 arcse for 1.1-m images and 0.92 arcsec for 0.8-m images.

\subsection{CCD Observations and Reductions}

Earlier studies of Hartley 2 had concluded that it has a small, "hyperactive" nucleus (e.g., Groussin et al. 2004; Lisse et al. 2009), so our imaging program focused on coma morphology rather than direct nucleus studies. Throughout the apparition, we regularly monitored the comet in broadband $\mathrm{R}$ and narrowband $\mathrm{CN}$ filters, with occasional narrowband blue continuum (BC) images as well. On photometric nights we used additional HB narrowband filters, increasing the variety and frequency as the comet brightened and shorter

exposure times were possible. Images using an $\mathrm{H}_{2} \mathrm{O}^{+}$filter were only obtained on 2010 November 4, in support of concurrent Chandra observations being conducted by Lisse et al. (2012); they are not discussed further in this paper. See Table 1 for a list of the filters used on a given night. Exposure times varied by filter and with the comet's brightness, but ranged from as long as $900 \mathrm{~s}$ (OH in September) to as short as $30 \mathrm{~s}$ ( $\mathrm{R}$ in November). Most R, 
$\mathrm{OH}$, and $\mathrm{CN}$ images were obtained in sets of 3-10 images while most images in other filters were obtained as single images. All 1.1-m images were guided at the comet's rate of motion while all 0.8-m images were tracked at the comet's rate of motion (the 0.8-m does not have guiding capability). The HB filters are parfocal and since the primary goal of the imaging campaign was gas coma morphology, we focused for these filters rather than for the R-band.

Our entire Hartley 2 campaign coincided with a separate effort to measure the nucleus lightcurve of 10P/Tempel 2 (Knight et al. 2012). As Tempel 2's period is known precisely, and is near $9 \mathrm{hr}$ and changing by $\sim+0.005 \mathrm{hr}$ per orbit (Knight et al. 2011), we required regular monitoring of its lightcurve over many months to detect a change in the period. Fortunately, Tempel 2 generally set as Hartley 2 was rising, but occasionally we split time on both objects. Thus, our temporal coverage of Hartley 2 was slightly less complete than it might have otherwise been, but this did not significantly affect any science results on Hartley 2 .

We removed the bias and applied a flat field following standard reduction techniques. We observed HB narrowband standard stars (Farnham et al.|2000) on all photometric nights. For these nights, we followed our standard photometric procedures (Farnham et al. 2000) to determine flux calibrations and process the narrowband images into pure gas and pure dust images. As discussed in Paper 1, there was not significant contamination from the dust continuum in CN images. This allowed us to make morphological assessments of CN images on non-photometric nights (when we could not generate fully "decontaminated" images). We normally only observe additional species on photometric nights, but obtained images in other filters despite non-photometric conditions on November 4 in support of the EPOXI flyby.

We centroided by fitting a two-dimensional Gaussian to the apparent photocenter of each image. As discussed in Paper 1, the jets observed in Hartley 2 can pull the centroid away from the nucleus. However, the central condensation generally dominated over any coma features and we estimate that our centroids are accurate to better than 1.5 arcsec. We investigated various enhancement techniques (e.g., Schleicher and Woodney 2003) but utilized division by an azimuthal median profile for all images shown in this paper.

\subsection{Photometer Observations and Reductions}

An observational set for photometry consisted of five gas filters $\left(\mathrm{OH}, \mathrm{NH}, \mathrm{CN}, \mathrm{C}_{3}\right.$, and $\mathrm{C}_{2}$ ) along with two (3650 and $4845 \AA$ ) or three $(3448,4450$, and $5260 \AA$ ) continuum filters associated with the IHW or HB filter sets, respectively. Three observational sets were 
obtained in 1991, four sets in 1997/98, and 55 sets in 2010/11 in support of EPOXI, with multiple sets often obtained on a single night. Photometer entrance aperture diameters ranged between 24 and 156 arcsec while projected aperture radii ranged from as small as $1950 \mathrm{~km}$ near perigee in 2010 October to as large as 47,900 km, with a median radius of $10,500 \mathrm{~km}$.

Our standard procedures were used in data acquisition and basic reductions (see A'Hearn et al. 1995), with appropriate changes associated with the newer HB filters (cf. Farnham et al. 2000); and improved decontamination for the IHW filters (cf. Farnham and Schleicher 2005). Gas fluorescence efficiencies that vary with heliocentric velocity are listed in Table 2, sources are given in Schleicher and Bair (2011). To extrapolate the resulting column abundances to total coma abundances, a standard Haser model was applied, using the scalelengths given in A'Hearn et al. (1995), and then gas production rates $(Q)$ were computed by dividing the total abundances by the assumed daughter lifetimes. Finally, water production rates were computed from the $\mathrm{OH}$ results using our empirical conversion (Cochran and Schleicher 1993; also see Schleicher et al. 1998). To quantify the dust, we continue to use the quantity $A(\theta) f \rho$ and, as appropriate, we have now applied a phase correction to the phase angle, $\theta$, listed in Table 2 (see Schleicher and Bair 2011) to obtain $A\left(0^{\circ}\right) f \rho$. One-sigma uncertainties based on the photon statistics associated with each data point were computed for the resulting

$Q \mathrm{~s}$ and $A(\theta) f \rho \mathrm{s}$. These were generally fairly small except for early and late in the 2010/11 apparition when we were attempting to extend the temporal coverage as much as possible and Hartley 2 was quite faint.

\section{NARROWBAND PHOTOMETRY}

\subsection{The Photometry Data Set}

We first present our photometric results for the three apparitions for which we have data. In Table 3 we list emission band and continuum fluxes (as logarithms) along with the resulting gas column abundances, $\mathrm{M}(\rho)$, while the derived gas production rates and $A(\theta) f \rho$ values for each continuum point are given in Table 4 as well as the vectorial-equivalent water production rate in the rightmost column. Note that although the $1-\sigma$ uncertainties are unbalanced in log-space, we only list the "+" log value for clarity and to save space; the "-" values can be computed knowing that the percent uncertainties are balanced. 


\subsection{Temporal Behavior}

The derived production rates for each gas species and $A(\theta) f \rho$ for the green continuum are plotted as logarithms in Figure 1 as a function of time from perihelion. The two most obvious characteristics are that the earlier apparitions generally have higher production rates than the most recent apparition and that all species exhibit a bulk asymmetry about perihelion, with values higher after but by varying amounts. As is usual for Jupiter-family comets (cf. A'Hearn et al. 1995), the heliocentric distance dependancies for the gas species $\left(\log Q\right.$ vs $\left.\log r_{\mathrm{H}}\right)$ are significantly steeper than a canonical $r_{\mathrm{H}}{ }^{-2}$ (or $r_{\mathrm{H}}{ }^{-2.5}$ if adjusting for gas outflow velocities). Based solely on the 2010/11 apparition, the slopes for the carbon-bearing species range from -3.4 to -4.1 before perihelion and from -3.2 to -3.5 after, while $\mathrm{OH}$ is -4.6 prior to and -4.0 after, and $\mathrm{NH}$ has an uncertain value of -7.1 before and -4.9 after perihelion (see Table 5).

The $r_{\mathrm{H}}$-dependancy for the dust, $A f \rho$, before perihelion is quite similar to those exhibited by the carbon-bearing species, especially if one normalizes to $0^{\circ}$ phase angle. Specifically, the three continuum points have values for the slopes from -2.8 to -3.6 (self-consistent given the large uncertainties) when using $A(\theta) f \rho$ while somewhat higher values from -3.4 to -4.1 using $A\left(0^{\circ}\right) f \rho$. Although the adjustment for phase angle has a larger effect after perihelion - going from -1.0 to -1.2 without adjustment to -1.7 to -1.9 when normalized - as seen in other comets, including 19P/Borrelly (Schleicher et al. 2003) and 67P/ChuryumovGerasimenko (Schleicher 2006), the $r_{\mathrm{H}}$-dependancies for dust are much shallower than that of the gas species following perihelion. As we suggested in Borrelly's case, the very shallow slope after perihelion is likely due to the release of larger, very slow moving grains near perihelion and peak water production, and these grains remain in the inner coma far longer than the more typical micron-sized dust particles. Note that Af $\rho$ also exhibits the usual trend with aperture size, where larger apertures yield smaller values, indicating that the dust spatial distribution with projected distance from the nucleus is steeper than the canonical $1 / \rho$. Since our projected aperture sizes were generally larger when the comet was at larger heliocentric distances, the $r_{\mathrm{H}}$-dependences just-discussed would have been even shallower had an adjustment for aperture size been performed, thereby magnifying the post-perihelion effect.

Returning to the earlier apparitions of the 1990s, we first note that all observational sets from 1997/98 are higher than corresponding data from 2010 except for the sole continuum

point after perihelion that can be explained by a combination of aperture and phase angle effects. The measured offset between these two apparitions ranges between about a factor of 1.5 and 2.0 among all species, far more than can be explained by the change in solar flux caused by the increase in perihelion distance from 1.032 AU in 1997 to 1.059 AU in 
2010. While our data are even sparser in 1991, with only three data sets on two nights, the measured offset is much greater, corresponding to a factor of 3-4 as compared to 2010. Again, this is far greater than expected due to solar illumination - perihelion was $0.953 \mathrm{AU}$ in 1991, resulting in only a $25 \%$ difference. To examine this further, we next intercompare a variety of water measurements from all three apparitions.

\subsection{Water Production}

As indicated in Section 2.3, we can compute vectorial-equivalent water production rates from our Haser-model $\mathrm{OH}$ production rates, and we plot these in Figure 2. We also overlay all other published water production values, including when available the uncertainties. We were surprised at the large amount of dispersion, even when only examining one apparition at a time. While some of this dispersion is likely due to differences in modeling parameters, some due to species-related issues (data are from direct water measurements in the IR, $\mathrm{OH}$ in the near-UV and radio, and $\mathrm{H}$ in the far-UV), and some due to varying dilutions by older material associated with an extremely large range of effective aperture sizes for the measurements, there remains a great deal of variability within some individual data sets, especially as compared to the apparent smooth curve with time evident in our own data.

Looking in detail first at the 2010/11 apparition, the variability seen in the most comprehensive data set from $S O H O /$ SWAN (Combi et al. $2011 \mathrm{a})$ is sometimes very small $(\Delta \mathrm{T}=$ -27 to -10 day) but other times more than a factor of two in only a few days. And while it first appears that our data are systematically higher than that from SOHO/SWAN, a closer examination reveals excellent agreement when data were obtained near-concurrently, such as at $\Delta \mathrm{T}$ of $-28 /-27$ day, +19 day, and +46 day (where the $S O H O /$ SWAN point is nearly invisible due to the overlap). Other data sets, such as Keck (Mumma et al. 2011), also suggest considerable short-term variability during some intervals in the apparition. Based on the ensemble of data, we conclude that our own smooth curve during 2010/11 was partly caused by having only one successful night of photometry on the majority of our observing runs, due to a combination of relatively poor weather and our focus towards obtaining imaging data (Section 4). Another factor is simple random chance; for instance our data sets from Nov 16 (+19 days) and Dec 13 (+46 days) are in complete agreement with other data on those nights. However, in neither case are these data similar in value to data obtained during the prior $\sim 10$ days.

Turning next to 1997/98, SOHO/SWAN (Combi et al. 2011b) again provides the most complete record, with one interval after perihelion showing a long steady decline while at other times much larger variability is evident. Also, in 1997, SOHO/SWAN measurements 
are systematically higher than ours, opposite of the case in 2010, but our two pre-perihelion data points each occur at minima of the $S O H O /$ SWAN data set. When looked at together, it becomes clear that there was a much greater drop in production rates between 1997 and 2010 , by about a factor of 3, than our data alone suggested. By extension, this would also indicate a smaller change took place between 1991 and 1997, and this is consistent with the only other published data point from 1991 (HST; Weaver et al. 1994), along with an unpublished IUE measurement taken within the week that was stated by Weaver et al. to be similar in value. Allowing for the change in perihelion distances by comparing the 1991 data with an extrapolated heliocentric distance trend post-perihelion, our 1991 data are about $50 \%$ higher while the HST (and IUE) points are nearly 90\% higher than 1997 . While the sparseness of the 1991 data and the large variability that is expected to be present make this result somewhat uncertain, the data are suggestive that water production rates were $\sim 70 \%$ greater in 1991 than in 1997.

From these water data, we conclude that there was a large $(\sim 3 \times)$ decrease in water vaporization between 1997 and 2010 (an interval of two orbits), and a smaller $(\sim 1.7 \times$ ) decrease between 1991 and 1997 (a single orbit), which implies a relatively consistent drop of about $40 \%$ from one apparition to the next. There is also considerable evidence for a stochastic amount of variability in water production as a function of time. There were intervals when variability was small, smooth, and exhibited trends consistent with the change in distance from the Sun, and other times when variability was far larger and apparently sporadic. Another striking comparison is the opposite trends evident in the SOHO data sets in the 3-4 weeks immediately after perihelion in 1997 versus 2010. Given the evidence from the EPOXI mission that there were several isolated source regions on the nucleus, coupled with evidence for a complex rotational state and a changing rotational period of at least one of the rotational components, we conclude that at least one source region experiences a very complicated solar illumination from a combination of these rotational effects. We further suggest that the intervals of smooth trends interrupted by intervals of much larger variability may be directly associated with the beat between the two periods, and how this beat changed with the changing period(s).

The absolute values of the water production can also yield a nominal surface area of vaporization. Our values in 2010 near perihelion and the EPOXI encounter imply an active area of $3-4 \mathrm{~km}^{2}$, just below the measured nucleus surface area of $\sim 4.2 \mathrm{~km}^{2}$ (based on a mean radius of $0.58 \pm 0.02 \mathrm{~km}$ from A'Hearn et al. 2011). Note that far more surface area than exists on the nucleus would have been required to produce the amount of water detected in the 1990s. The significance of this will be returned to in Section 5 . 


\subsection{Composition}

The composition of Hartley 2, based on the abundance ratios of the minor gas species computed using the ratios of their respective production rates with respect to $\mathrm{OH}$, are given in Table 5. Here we also provide our standard uncertainties, the $\sigma$ of the data, which we have tabulated in our previous single comet papers, along with the $\sigma$ of the mean. The former describes the dispersion of the data, due to observational errors, modeling effects, and intrinsic variations in the comet itself, while the latter better describes how well each mean ratio is determined.

Hartley 2's composition clearly places it in the middle of the "typical" classification from A'Hearn et al. (1995), consistent with our result from the 1991 apparition that was included in A'Hearn et al. Using this same classification scheme, Lara et al. (2011) find the same result from their spectroscopic observations. The comet is also classified as "typical" by Fink (2009) using his own definition of compositional classes.

Determining a single mean value for the dust-to-gas ratio is more problematic for several reasons, including the trend in $A f \rho$ values with aperture size, the much shallower $r_{\mathrm{H}^{-}}$ dependence following perihelion, and phase angle effects. Since the first two issues are incorporated in our calculation of the uncertainties of the data for the dust-to-gas ratio, we make no further adjustments. Regarding phase angle effects, we compute both an unadjusted value, i.e. $A(\theta) f \rho / Q(\mathrm{OH})$, for direct comparison to other comets in A'Hearn et al. (1995), along with a normalized value to $0^{\circ}$ phase angle. Our results for the green continuum were -25.84 and -25.44 , respectively, corresponding to a relatively low dust-to-gas ratio, especially as compared to other Jupiter-family comets. Finally, based on the three continuum points we measure, the dust is nearly grey in color, with reddening of less than $10 \%$ per $1000 \AA$ through most of the apparition. However, at the end of October, when our projected apertures were smallest, the color in the UV was as high as 30\% per $1000 \AA$. This result is consistent with that by Lara et al. (2011) who detect a stronger reddening in the inner-most coma, but note that the reddening decreases with distance and also varies with direction in the coma and that the dust is blue in some locations.

\section{COMA MORPHOLOGY}

\subsection{Review of CN Morphology}

Paper 1 focused on Hartley 2's CN coma morphology. We summarize the relevant results here to place the morphology observed in $\mathrm{OH}, \mathrm{NH}, \mathrm{C}_{3}, \mathrm{C}_{2}$, and dust into context. We 
observed two CN features, generally towards the north and south. In August and September only the northern feature was seen, and had the appearance of a nearly face-on spiral. In October, November, and December the morphology was roughly an hourglass shape, with outward motion visible and the relative brightnesses of the northern and southern features varying. In January the signal-to-noise was poor and only the southern feature was detected. The CN morphology varied smoothly during a night and similar, but not identical, morphology was seen on subsequent nights. The similar morphology allowed us to estimate a rotation period during the August to November runs, and the period increased by $\sim 2 \mathrm{hr}$ (from $\sim 16.7 \mathrm{hr}$ to $\sim 18.7 \mathrm{hr}$ ) during this time. The differences in morphology implied the existence of small deviations from a principal axis rotation. The morphology repeated much better $3,6,9 \ldots$ cycles apart than it did $1,2,4,5 \ldots$ cycles apart, implying a component rotation period $\sim 3 \times$ the "primary" rotation period. Similar morphology was reported by Samarasinha et al. (2011), Lara et al. (2011), and Waniak et al. (2012), and the changing rotation period and non-principal axis rotation were confirmed by other observers (cf. Jehin et al. 2010; A'Hearn et al. 2011; Drahus et al. 2011; Samarasinha et al. 2011).

\subsection{Coma Morphology of $\mathrm{C}_{3}, \mathrm{C}_{2}, \mathrm{OH}$, and $\mathrm{NH}$}

$\mathrm{CN}$ is generally the gas species of choice for investigations of gas coma features with narrowband filters because it is vastly brighter than $\mathrm{OH}$ or $\mathrm{NH}$ and has a better contrast relative to the dust than $\mathrm{C}_{2}, \mathrm{C}_{3}$, or $\mathrm{NH}$. $\mathrm{CN}$ jets were first detected in the coma of $1 \mathrm{P} / \mathrm{Halley}$ A'Hearn et al. 1986) and jets and/or fans have now been detected in numerous other comets, e.g., C/1995 O1 Hale-Bopp (Woodney et al. 2002), C/2004 Q2 Machholz (Farnham et al. 2007), 8P/Tuttle (Waniak et al. 2009), and C/2007 N3 Lulin (Knight and Schleicher 2009). The coma morphology of the other gas species has only been published for a few very bright comets, e.g., 1P/Halley Cosmovici et al. 1988; Schulz and A'Hearn 1995) and C/1995 O1 Hale-Bopp (Lederer et al. 1997). Hartley 2's very small geocentric distance during the 2010/11 apparition allowed us to observe it with unusually high spatial resolution, making a multiwavelength study of coma morphology highly desirable. As listed in Table 1, we obtained $\mathrm{C}_{2}$ and $\mathrm{C}_{3}$ images on photometric nights from August through November, $\mathrm{OH}$ images on photometric nights from September through November, and NH images on photometric nights in October and November (although the lone October NH image is unusable due to a tracking error). The comet was easily detectable in all of these raw images and after removal of dust contamination. However, sufficient signal-to-noise to allow meaningful analysis of the enhanced images was only possible in October and November for species other than CN. We show $\mathrm{CN}, \mathrm{C}_{3}, \mathrm{C}_{2}, \mathrm{OH}, \mathrm{NH}$, and dust on November 2 and 3 in Figure 3 ; comparable data were obtained on October 16 and November 7 but are not shown. 
As shown in Figure 3, the $\mathrm{C}_{3}$ and $\mathrm{C}_{2}$ morphology looked generally similar to the $\mathrm{CN}$ morphology. On November 2, all three species exhibited an hourglass-like shape, with a stronger feature to the north and a fainter feature to the south; the southern feature can be seen leaving the nucleus towards the southwest. On November 3 , the $\mathrm{CN}, \mathrm{C}_{3}$, and $\mathrm{C}_{2}$ showed a somewhat different hourglass shape, with the the southern feature much brighter relative to the northern feature than on November 2. All three species were much fainter along the sun-tail line (roughly east-west) than along the axis of the hourglass feature. The CN signal was typically lowest in the tailward direction, whereas the $\mathrm{C}_{3}$ and $\mathrm{C}_{2}$ signals were typically slightly fainter in the sunward direction. The overall bulk brightness of $\mathrm{C}_{3}$ and $\mathrm{C}_{2}$ varied in correlation with the bulk CN brightness, e.g., generally brighter to the north on November 2 and generally brighter to the south on November 3.

In order to quantify the differences between the gas species, we show their spatial profiles in Figure 4. We plot the flux (as measured on decontaminated, but not enhanced, images) on November 2 as a function of distance from the nucleus in $10^{\circ}$ wide wedges centered along position angles (PAs) of: $10^{\circ}$ (perpendicular to the sunward direction, to the north), $100^{\circ}$ (the sunward direction), $190^{\circ}$ (perpendicular to the sunward direction, to the south), and $280^{\circ}$ (the tailward direction). These profiles extend to the edges of the images shown in Figure 3. The inner $\sim 800 \mathrm{~km}$ are not plotted as there are very few pixels in each wedge at these distances, causing small fluctuations to be exaggerated. The northern and southern CN profiles were $\sim 20 \%$ and $\sim 10 \%$ brighter than the sunward profile, respectively. The CN tailward profile was roughly the same brightness as the sunward profile. The $\mathrm{C}_{3}$ and $\mathrm{C}_{2}$ profiles were both brightest to the north, followed by the south, then tailward, and finally sunward. The north, south, and tailward profiles were 10-30\% brighter than the sunward profile. Note that these brightnesses are along the line of sight and are products of projection effects, each species' parentage, and excess velocities acquired. Thus, brightness enhancements in a particular direction relative to the sunward direction (which did not have any obvious jets) are likely only lower limits, and the actual ratio of the material originating from the nucleus in each direction may be substantially higher.

Dividing the $\mathrm{C}_{2}$ and $\mathrm{C}_{3}$ images by the $\mathrm{CN}$ images revealed minimal differences in their morphologies, with the primary feature (the hourglass shape) disappearing in quotient images. As is clearly evident in Figure 4, the $\mathrm{C}_{3}$ does not extend as far as the other gas species. This was partially due to the higher signal-to-noise in $\mathrm{CN}$ and $\mathrm{C}_{2}$ as compared to $\mathrm{C}_{3}$, however, it was mostly due to the differing lifetimes of the species and their respective parentages; $\mathrm{C}_{3}$ and its parents have much shorter lifetimes than either $\mathrm{C}_{2}$ or $\mathrm{CN}$ and their respective parents (cf. A'Hearn et al. 1995). $\mathrm{C}_{2}$ appeared quite similar to $\mathrm{CN}$ but was more diffuse because it has multiple parents and grandparents, whereas $\mathrm{CN}$ is primarily a daughter species. The multiple parentages of $\mathrm{C}_{2}$ cause its radial profile to be flatter than the profiles 
of either $\mathrm{CN}$ or $\mathrm{C}_{3}$.

Owing to the shorter observing window when the comet was brightest and the desire to acquire images in a large number of filters, we usually only acquired one or two $\mathrm{C}_{2}$ or $\mathrm{C}_{3}$ images per night. Thus, we cannot investigate how the morphology of these species compares as a function of rotation period. However, on every night in which we saw $\mathrm{CN}, \mathrm{C}_{3}$, and $\mathrm{C}_{2}$ with sufficient signal-to-noise to discern varitions in the coma, the morphologies appeared to be completely consistent except for the parentage and lifetime effects discussed above. This leads us to conclude that the $\mathrm{C}_{3}$ and $\mathrm{C}_{2}$ species exhibit the same rotational and seasonal morphology as $\mathrm{CN}$ and therefore originate from the same source region(s) on the surface.

Near the nucleus, the $\mathrm{NH}$ displayed some of the hourglass morphology of $\mathrm{CN}, \mathrm{C}_{3}$, and $\mathrm{C}_{2}$. The features appeared to originate from separate northern and southern sources, although the distinction between the features in the tailward direction was less obvious than for $\mathrm{CN}$, $\mathrm{C}_{3}$, or $\mathrm{C}_{2}$. The relative brightness of the northern and southern features varied in correlation with changes in the brightness of these features in CN. NH had a strong asymmetry in brightness of the sunward and tailward hemispheres, with the tailward hemisphere always being substantially brighter. The bulk brightness of the NH images displayed little variation from night to night. The radial profiles demonstrate the extent of the tailward brightness enhancement, as the north, west, and south profiles all remained at least $20 \%$ higher than the eastern (sunward) profile to the edge of the images displayed in Figure 3 . As with $\mathrm{C}_{2}$, the $\mathrm{NH}$ radial profiles were rather flat due to its being primarily a granddaughter species. We used the photometry to determine the amount of reddening to apply in order to properly remove the underlying continuum from the NH. However, it is likely that the continuum was somewhat over-removed in the innermost coma $(<3000 \mathrm{~km})$ because the dust was increasingly reddened at progressively smaller distances from the nucleus, resulting in the unusually flat profile.

The morphology most different from $\mathrm{CN}$ was $\mathrm{OH}$. The $\mathrm{OH}$ morphology near the nucleus showed only hints of the hourglass shape. Unlike the other gas species, the feature did not have distinct northern and southern components, but was essentially continuous from the north through the west (tailward) to the south. The relative brightnesses of the north and south features of the hourglass vary much less than the other gas species. Like NH, the tailward hemisphere was much brighter than the sunward hemisphere. Furthermore, at larger distances the $\mathrm{OH}$ was brighter toward the north on all four nights in November, and the bulk brightness did not vary significantly from night to night. The tailward profile was $\sim 35 \%$ higher than the sunward profile, while the northern and southern profiles were $20-25 \%$ higher than the sunward profile.

The striking differences between the coma morphologies of $\mathrm{OH}$ and $\mathrm{CN}$ was seen on 
every night in which we have usable $\mathrm{OH}$ data, as shown in Figure 5. In each of these images the $\mathrm{OH}$ signal near the nucleus showed hints of the hourglass shape seen in CN (although the faint $\mathrm{CN}$ feature near the nucleus and towards the southeast on November 4 is absent in $\mathrm{OH}$ ). The tailward $\mathrm{OH}$ hemisphere was always much brighter than the sunward hemisphere, and the dust tail (BC) almost perfectly bisected the $\mathrm{OH}$ distribution in all images, including October 16 when the geometry was substantially different. The hemispheric brightness asymmetry and the lack of variation in the bulk brightness from night to night suggest that there is a smearing across rotational phase in the $\mathrm{OH}$ signal, and that the changing viewing geometry was primarily responsible for the changes in $\mathrm{OH}$ morphology between October and November.

As with $\mathrm{C}_{2}$ and $\mathrm{C}_{3}$, we typically only acquired one or two sets of $\mathrm{OH}$ and $\mathrm{NH}$ on a given night so we cannot determine the morphology throughout an entire rotation cycle. However, during each night in which we observed $\mathrm{OH}$ and $\mathrm{NH}$, their bulk coma morphologies looked similar - concentrated in the tailward hemisphere - and distinctly different than $\mathrm{CN}, \mathrm{C}_{2}$, or $\mathrm{C}_{3}$. The consequences of these determinations will be returned to in Section 5 .

\subsection{Dust Morphology}

The dust morphology of Hartley 2 was dominated by the tail throughout the apparition. Figure 6] shows enhanced R-band images monthly from 2010 August through 2011 January. This same shape was seen, albeit with lower signal-to-noise, in the narrowband continuum filters: UV, blue, green, and red. In all cases the dominant feature was roughly straight and in the anti-sunward direction (the PA of the Sun is labeled on each panel). It does not vary with rotational phase and is consistent with the expected position of the dust tail, confirming that it is the dust tail. Overall, the brightness of the dust systematically decreased as a function of PA from the tailward direction to the sunward direction. Radial profiles of the four fundamental directions are shown in Figure 4.

We detected a very faint sunward facing dust jet near the nucleus, as also reported by Lara et al. (2011) and Mueller et al. (2012). While difficult to see in Figures 3 and 5 , it can be seen in the BC profile in Figure 4, as the sunward profile is slightly higher than the northern or southern profiles out to $\sim 1600 \mathrm{~km}$. The jet was nearly radial and showed minimal change in shape, position angle, or extent during the course of a night, but varied somewhat from night to night. We show examples of the jet near the start and end of the night in enhanced continuum images on 2010 November 2, 3, 4, and 7 in Figure 7. The jet's appearance was relatively similar on November 2 and 7 , pointing nearly due east at PAs of $\sim 95^{\circ}$ and $\sim 100^{\circ}$, respectively. It had a slightly different appearance on November 3 and 
4, when it was to the southeast at PAs of $\sim 115^{\circ}$ and $\sim 125^{\circ}$, and extended farther than on November 2 or 7 . At its maximum extent (on November 3), the dust jet was visible to 2025 arcsec from the nucleus, although signal-to-noise and our enhancement techniques may have prevented us from seeing it extend farther. Hints of the dust jet were apparent in the October 16 and 17 continuum images, but the extent was much smaller $(<10$ arcsec) than in early November despite better signal-to-noise. The lack of a firm detection in October may have been because the jet was inactive or it may simply have been due to projection effects. The dust jet was not detectable in our data from other months, presumably due to the significantly lower signal-to-noise and larger geocentric distance as compared to 2010 October and November. At no time did we observe a corresponding gas feature.

Surprisingly, the dust jet was easier to detect in our narrowband dust images - blue continuum (BC), green continuum (GC), and red continuum (RC) - than in the broadband $\mathrm{R}$ images. We applied several additional enhancement techniques to the R-band images in an effort to study the dust jet with more temporal resolution than the continuum images offered. However, we could not consistently detect the dust jet in consecutive R-band images despite the R-band images having a higher signal-to-noise than the continuum images. All of our Hartley 2 observations were focused for the HB narrowband filters, resulting in a typical R-band FWHM of $\sim 6$ arcsec. Given the faintness of the dust jet, this R-band defocusing appears to have been sufficient to obscure the dust jet. Since, as was the case for $\mathrm{C}_{3}, \mathrm{C}_{2}$, $\mathrm{OH}$, and $\mathrm{NH}$, we only obtained narrowband dust images occasionally, we do not have full rotational coverage. Thus we cannot determine how the dust jet varied with rotation, but our limited data exhibit a slower rate of change than was exhibited by the gas jet(s).

\section{DISCUSSION}

The coma morphologies varied across the five gas species we observed. At one extreme was CN whose morphology was dominated by an hourglass shaped feature. At the opposite extreme was $\mathrm{OH}$, whose morphology appeared to be an amalgamation of the $\mathrm{CN}$ hourglass

feature and the tail-dominated dust. In between these extremes were $\mathrm{C}_{3}$ and $\mathrm{C}_{2}$, whose morphologies were generally quite similar to the $\mathrm{CN}$, and the $\mathrm{NH}$, which appeared to be intermediate to the $\mathrm{CN}$ and the $\mathrm{OH}$.

The presence of the hourglass shape exhibited clearly in $\mathrm{CN}, \mathrm{C}_{3}$, and $\mathrm{C}_{2}$, and to a lesser extent by $\mathrm{NH}$ and $\mathrm{OH}$ suggests that all five species originate from the same source region(s) on the nucleus. This is supported by the observation that the relative brightness of the northern and southern features in $\mathrm{CN}, \mathrm{C}_{3}, \mathrm{C}_{2}$, and $\mathrm{NH}$ vary in concert, and also that the $\mathrm{CN}, \mathrm{C}_{3}$, and $\mathrm{C}_{2}$ morphologies are in agreement from night to night. The relative brightness 
of the $\mathrm{OH}$ hourglass features did not vary as much as the other gas species, and $\mathrm{OH}$ also exhibited more near-nucleus coma signal in the tailward direction than the other species.

The hemispheric asymmetry of $\mathrm{OH}$ and $\mathrm{NH}$ as well as the relative uniformity of their bulk brightness (after enhancement) suggest that a substantial fraction of the $\mathrm{OH}$ and $\mathrm{NH}$ is derived from small icy grains which survived long enough to be subject to radiation pressure and swept tailward. The infilling of $\mathrm{OH}$ and $\mathrm{NH}$ throughout the tailward hemisphere is likely due to the velocities in random directions acquired by the parent molecules as they leave the grains. EPOXI observed numerous individual chunks up to $\sim 20 \mathrm{~cm}$ in radius within $30 \mathrm{~km}$ of the nucleus (A'Hearn et al. 2011) and Harmon et al. (2011) reported a significant population of large $(>\mathrm{cm})$ dust grains near the nucleus. A'Hearn et al. suggest that these large grains break up into $\sim 1 \mu \mathrm{m}$ solid grains, with the smaller grains providing the surface area necessary to explain Hartley 2's "hyperactivity." The bulk brightness enhancement of the $\mathrm{OH}$ and $\mathrm{NH}$ in the tailward hemisphere supports this model, with the micron-sized grains containing the water and ammonia that eventually produced the $\mathrm{OH}$ and $\mathrm{NH}$.

EPOXI revealed distinctly different terrain on the nucleus, with a smooth "waist" connecting two rougher lobes (A'Hearn et al. 2011). A'Hearn et al. determined that different material was coming from these two regions, with $\mathrm{H}_{2} \mathrm{O}$ vapor coming primarily from the waist and $\mathrm{CO}_{2}, \mathrm{H}_{2} \mathrm{O}$ ice, and organics coming primarily from an end. Thus, it is likely that the $\mathrm{CN}, \mathrm{C}_{3}, \mathrm{C}_{2}, \mathrm{OH}$, and $\mathrm{NH}$ originate near an end of a lobe, presumably from one or more of the many active regions observed by EPOXI. Another very strong constraint is the observation from our photometry that the ratios of the minor species to water are normal; therefore, the vast majority of all of our observed species must be originating at the same time and location. The differences in the bulk morphology between the $\mathrm{NH}$ and $\mathrm{OH}$ and the $\mathrm{CN}, \mathrm{C}_{3}$, and $\mathrm{C}_{2}$ can be explained if the icy grains containing $\mathrm{OH}$ and $\mathrm{NH}$ are separated from the parents/grandparents of $\mathrm{CN}, \mathrm{C}_{3}$, and $\mathrm{C}_{2}$ (either as smaller grains or simply as vapor) soon after being released from the nucleus.

Such a scenario suggests that there may have been an intrinsic difference in the protosolar grains out of which Hartley 2 formed, with the parents of $\mathrm{OH}$ and $\mathrm{NH}$ (primarily water and ammonia, respectively) preferentially being contained in larger grains (and possibly intermixed as "dirty" ice) while the parents of $\mathrm{CN}, \mathrm{C}_{2}$, and $\mathrm{C}_{3}$ were contained in smaller grains or were deposited primarily on the surface of larger grains. It is somewhat troubling that such a difference in grain compositions has never been detected in other comets (especially in light of Hartley 2's "typical" composition), but given the relative uniqueness of Hartley 2's "hyperactivity," it is not surprising that it may have exhibited other unusual properties; note that no other comet showing hyperactivity has been visited by a spacecraft. The mechanism for lifting large grains, suggested to be $\mathrm{CO}_{2}$ (A'Hearn et al. 2011), may be vigorous enough 
to lift off large chunks of the surface in toto, likely resulting in a considerably different removal of material than the canonical model of cometary activity which envisions gas leaving through pores in the surface and entraining small bits of dust in the process. Conversely, very few comets have been imaged in $\mathrm{NH}$ and/or $\mathrm{OH}$ owing to their low signal and high atmospheric extinction. Thus, the different coma morphologies of $\mathrm{OH}$ and $\mathrm{NH}$ compared with the carbon bearing species may not, in fact, be unusual.

As discussed in Section 4.3, the dust jet showed little variation during a night but changed somewhat from night to night, always being in the same general direction. These two points are consistent with the apparent non-principal axis rotation of the nucleus (cf. A'Hearn et al. 2011; Samarasinha et al. 2011; Paper 1). The first point suggests that the source of the jet might be located near the comet's total angular momentum vector, the axis around which the nucleus was apparently "precessing" with a period near $18.3 \mathrm{hr}$ at the time (cf. A'Hearn et al. 2011), resulting in little change in the morphology over a night. The second point could be caused by Hartley 2's longer "rotation" period of $\sim 55 \mathrm{hr}$ at the time of the observations. This would not have much effect on the jet's appearance over the course of one night (only $\sim 10 \%$ of a $55 \mathrm{hr}$ period), but would cause changes from night to night (when the rotational phase had changed by roughly $45 \%$ ).

The lack of a gas feature corresponding to the dust jet implies that the dust is lifted from the surface by some other volatile. The most likely candidates are CO and, more likely, $\mathrm{CO}_{2}$, which A'Hearn et al. (2011) concluded drives activity on Hartley 2. Presumably this source has little to no $\mathrm{CN}, \mathrm{C}_{3}, \mathrm{C}_{2}$, $\mathrm{OH}$, or $\mathrm{NH}$ since no corresponding gas jet was seen; their absence implies that the source has a different composition than the source(s) of the hourglass features and suggests some heterogeneity of the Hartley 2 nucleus. We do not see evidence of radiation pressure affecting the dust jet, which implies we are primarily seeing large dust grains. The velocity dispersion of large grains would be expected to mask much of the rotational signature of the dust jets. Since we do not see any evidence of dust jets near the locations of the hourglass features in the gas, we infer that the gas jet(s) do not have substantial quantities of large grains. Instead, the jet(s) likely lifts small grains which are rapidly pushed tailward; a similar population of small dust grains subject to radiation pressure is likely present in the dust jet and goes similarly undetected.

We next turn to the observation (Section 3.4) that the production rates have trended downwards steadily from 1991 to 1997/98 to 2010/11 even after accounting for the increase in perihelion distance between apparitions. We speculate that this may be due to the relative youth of the nucleus. In the early twentieth century, Hartley 2 had a perihelion distance $(q)$ near $2 \mathrm{AU}$ and an orbital period near 9 yr. Close approaches to Jupiter in 1947 and 1971 caused a drop in the perihelion distance, putting Hartley 2 in an orbit reaching $\sim 1$ AU. If the 
primary driver of activity was something more volatile than $\mathrm{H}_{2} \mathrm{O}$ such as $\mathrm{CO}_{2}$ as suggested by A'Hearn et al. (2011) (CO is less likely due to the extremely low abundance; Weaver et al. 2011), then the injection into a smaller- $q$ orbit may be rapidly depleting the $\mathrm{CO}_{2}$. Since vigorous activity is required to lift off large chunks of particles necessary to produce the "hyperactivity", a rapid decrease in the production of $\mathrm{CO}_{2}$ would cause a correspondingly large drop in the production rate of $\mathrm{H}_{2} \mathrm{O}$.

The factor of 1.5-3 drop in the production rates between 1997/98 and 2010/11 spanned two perihelion passages (we are not aware of any published production rate measurements from the 2004 apparition), allowing additional time for production from such areas to decrease. As there were no systematic surveys that would have been sensitive to this intrinsically faint comet on earlier, less favorable, apparitions, we cannot place meaningful constraints on the production rates prior to its discovery in 1986 . If a similar rate of decrease in production rate occurred between 1971 and 1991, Hartley 2 may have been as much as a factor of 8-10 more active in 1971 (when it was first perturbed into the current orbit) than in 2010/11.

An alternative, but less likely, explanation for the decreased production rates since 1997/98 may simply be that the illumination of active regions was different between the apparitions. Hartley 2 exhibited a rapid spin-down coupled with non-principal axis rotation during the 2010/11 apparition (cf. A'Hearn et al. 2011; Knight and Schleicher 2011; Samarasinha et al. 2011). It is possible that on previous orbits the interplay of the complex rotation and the apparently increasing and decreasing component periods (cf. the Support-

ing Online Materials from A'Hearn et al. 2011) could result in a different illumination of one or more active regions. This might include the Sun reaching a higher altitude, variations in topography causing less shadowing, the exposure of additional active regions, or the Sun remaining above an active region's local horizon longer. However, the nucleus' complex rotation state should minimize seasonal effects; unless the nucleus was very recently (since 1997/98) excited into the present non-principal axis rotation state, large changes in the production rates due entirely to geometry are considered unlikely.

\section{SUMMARY}

We have presented photometry and imaging of 103P/Hartley 2 obtained at Lowell Observatory (and one night at Perth Observatory) from 1991 through 2011. The photometry includes three apparitions as no data were obtained on the 2004 apparition. We find a secular decrease in brightness from the 1991 apparition to the 1997/98 apparition and then to the 2010/11 apparition and a signficant seasonal effect, with the comet reaching peak brightness 
$\sim 10$ days after perihelion and the rate of brightening steeper than the rate of fading. We find similar results when compiling published water production rates from the literature, consistent with about a $40 \%$ decrease each orbit. Hartley 2's composition is "typical," in agreement with the results of other investigators. We propose two scenarios for the relatively large decrease in production rates: that production rates are dropping rapidly owing to the rapid depletion of $\mathrm{CO}_{2}$ (due to the decrease in perihelion distance in the mid-twentieth century) or, less likely, that the "complex" nucleus rotation resulted in progressively less illumination reaching the primary active regions from 1991 to 1997/98 to 2010/11.

Our imaging covered the 2010/11 apparition and focused on the gas coma morphology. We previously reported on our extensive $\mathrm{CN}$ data set (Paper 1) and analyze here the morphology exhibited by other gas species $\left(\mathrm{OH}, \mathrm{NH}, \mathrm{C}_{3}\right.$, and $\left.\mathrm{C}_{2}\right)$ as well as the dust. We find that $\mathrm{C}_{3}$ and $\mathrm{C}_{2}$ exhibit coma morphology generally similar to what we previously reported for $\mathrm{CN}$, with an hourglass shape in October and November that followed the rotational changes exhibited by $\mathrm{CN}$, and little excess signal in the tailward direction. We conclude that differences between the $\mathrm{CN}, \mathrm{C}_{3}$, and $\mathrm{C}_{2}$ coma morphologies can be explained by their different lifetimes and parentages. The $\mathrm{OH}$ and $\mathrm{NH}$ coma morphologies differ from $\mathrm{CN}, \mathrm{C}_{3}$, and $\mathrm{C}_{2}$; while $\mathrm{OH}$ and $\mathrm{NH}$ show evidence of the hourglass shape near the nucleus, they are relatively uniform in brightness (after enhancement) throughout the tailward hemisphere, and the brightness in the tailward hemisphere does not vary appreciably with rotation. We conclude that $\mathrm{OH}$ and $\mathrm{NH}$ are produced from water and ammonia ices that were contained in small grains which shielded the ices long enough to be affected by radiation pressure and driven in the anti-sunward direction. We speculate on possible explanations for why the $\mathrm{OH}$ and $\mathrm{NH}$, but not the $\mathrm{CN}, \mathrm{C}_{3}$, or $\mathrm{C}_{2}$, behave in this manner, concluding that all five species most likely originate from the same source region(s) near the end of a lobe of the nucleus (where EPOXI saw significant active regions) but may have come from aggregates of different sized grains or grains and vapor. Lower velocities and/or a range of velocities associated with a variety of particle sizes would also naturally cause a dilution of the rotational signature of $\mathrm{OH}$ and $\mathrm{NH}$ as compared to that seen in the carbon bearing species. We detect the faint sunward-facing dust jet reported by Lara et al. (2011) and Mueller et al. (2012) in our continuum images. This jet is much smaller than the hourglass shaped gas feature and does not vary appreciably during a night, although it does vary from night to night. No corresponding gas feature was seen at the location of the dust jet, possibly implying that it is driven by $\mathrm{CO}_{2}$. The dust jet may originate from a source near the total angular momentum vector.

The EPOXI flyby and supporting observations of Hartley 2 have revealed that it is an unusual comet which is highly active relative to its nucleus size, has a population of large grains in the inner coma, and is in non-principal axis rotation with evolving component 
periods. As one of a small handful of comets to have been visited by a spacecraft, Hartley 2 represents a rare opportunity to link the macroscopic coma morphology and abundances observed remotely with the nucleus shape and active regions observed in situ via sophisticated modeling. The strongest constraints on such models are likely the direction of the nucleus' long axis as seen by EPOXI during the flyby (cf. A'Hearn et al. 2011) and the alignment of the rotational angular momentum at the time of the Arecibo observations (Harmon et al. 2011). However, any comprehensive jet modeling should also incorporate the constraints discussed here and in Paper 1. These include the morphological changes during a night (outward motion of the material and the sense of rotation), differences in morphology from night to night (due to the non-principal axis rotation), and evolution of the morphology from month to month (due to the changing viewing geometry). It is only by satisfying these varied constraints that a model can produce a coherent description of Comet Hartley 2.

\section{ACKNOWLEDGEMENTS}

We appreciate the work of Anita Cochran and Nalin Samarasinha in carefully refereeing this manuscript and making helpful suggestions to improve the paper. We are indebted to the following people for help in collecting data: Robert Millis (1991); Tony Farnham (1997/1998 Lowell data); Peter Birch (1997 Perth data); Len Bright, Brian Skiff, Larry Wasserman, and Edward Schwieterman (2010/2011 imaging data). We thank Allison Bair for help in producing Table 5 and for comparisons with preliminary results of the forthcoming comet database paper. Office space was generously provided for MMK by both the University of Maryland Department of Astronomy and Johns Hopkins University Applied Physics Laboratory while he conducted this work. This work has been supported by numerous NASA grants over the years, most recently NNX08AO42G and NNX09AB51G.

\section{REFERENCES}

A’Hearn, M. F. 1991. Photometry and Polarimetry Network, pp. 193.

A’Hearn, M. F., M. J. S. Belton, W. A. Delamere, L. M. Feaga, D. Hampton, J. Kissel, K. P. Klaasen, L. A. McFadden, K. J. Meech, H. J. Melosh, P. H. Schultz, J. M. Sunshine, P. C. Thomas, J. Veverka, D. D. Wellnitz, D. K. Yeomans, S. Besse, D. Bodewits, T. J. Bowling, B. T. Carcich, S. M. Collins, T. L. Farnham, O. Groussin, B. Hermalyn, M. S. Kelley, M. S. Kelley, J.-Y. Li, D. J. Lindler, C. M. Lisse, S. A. McLaughlin, F. Merlin, S. Protopapa, J. E. Richardson, and J. L. Williams 2011. EPOXI at Comet Hartley 2. Science 332, 1396-1400. 
A'Hearn, M. F., S. Hoban, P. V. Birch, C. Bowers, R. Martin, and D. A. Klinglesmith, III 1986. Cyanogen Jets in Comet Halley. Nature 324, 649-651.

A'Hearn, M. F., R. L. Millis, D. G. Schleicher, D. J. Osip, and P. V. Birch 1995. The Ensemble Properties of Comets: Results from Narrowband Photometry of 85 Comets, 1976-1992. Icarus 118, 223-270.

Cochran, A. L., and D. G. Schleicher 1993. Observational Constraints on the Lifetime of Cometary $\mathrm{H}_{2} \mathrm{O}$. Icarus 105, 235-253.

Colangeli, L., E. Epifani, J. R. Brucato, E. Bussoletti, C. de Sanctis, M. Fulle, V. Mennella, E. Palomba, P. Palumbo, and A. Rotundi 1999. Infrared Spectral Observations of Comet 103P/Hartley 2 by ISOPHOT. A $ध A$ 343, L87-L90.

Combi, M. R., J.-L. Bertaux, E. Quémerais, S. Ferron, and J. T. T. Mäkinen 2011a. Water Production by Comet 103P/Hartley 2 Observed with the SWAN Instrument on the SOHO Spacecraft. ApJ 734, L6.

Combi, M. R., Y. Lee, T. S. Patel, J. T. T. Mäkinen, J.-L. Bertaux, and E. Quémerais 2011b. SOHO/SWAN Observations of Short-period Spacecraft Target Comets. AJ 141, 128.

Cosmovici, C. B., G. Schwarz, W.-H. Ip, and P. Mack 1988. Gas and Dust Jets in the Inner Coma of Comet Halley. Nature 332, 705-709.

Crovisier, J., Colom, P., Biver, N., Bockelée-Morvan, D., \& Boissier, J. 2012, Icarus, this issue.

Dello Russo, N., R. J. Vervack, Jr., H. Kawakita, and H. Kobayashi 2010. Comet 103P/Hartley. IAU Circ. 9171.

Dello Russo, N., R. J. Vervack, Jr., C. M. Lisse, H. A. Weaver, H. Kawakita, H. Kobayashi, A. L. Cochran, W. M. Harris, A. J. McKay, N. Biver, D. Bockelée-Morvan, and J. Crovisier 2011. The Volatile Composition and Activity of Comet 103P/Hartley 2 During the EPOXI Closest Approach. ApJ 734, L8.

Drahus, M., D. Jewitt, A. Guilbert-Lepoutre, W. Waniak, J. Hoge, D. C. Lis, H. Yoshida, R. Peng, and A. Sievers 2011. Rotation State of Comet 103P/Hartley 2 from Radio Spectroscopy at $1 \mathrm{~mm}$. ApJ 734, L4.

Farnham, T. L., N. H. Samarasinha, B. E. A. Mueller, and M. M. Knight 2007. Cyanogen Jets and the Rotation State of Comet Machholz (C/2004 Q2). AJ 133, 2001-2007. 
Farnham, T. L., and D. G. Schleicher 2005. Physical and Compositional Studies of Comet 81P/Wild 2 at Multiple Apparitions. Icarus 173, 533-558.

Farnham, T. L., D. G. Schleicher, and M. F. A'Hearn 2000. The HB Narrowband Comet Filters: Standard Stars and Calibrations. Icarus 147, 180-204.

Fink, U. 2009. A Taxonomic Survey of Comet Composition 1985-2004 Using CCD Spectroscopy. Icarus 201, 311-334.

Groussin, O., P. Lamy, L. Jorda, and I. Toth 2004. The Nuclei of Comets 126P/IRAS and 103P/Hartley 2. A\&A 419, 375-383.

Harmon, J. K., M. C. Nolan, E. S. Howell, J. D. Giorgini, and P. A. Taylor 2011. Radar Observations of Comet 103P/Hartley 2. ApJ 734, L2.

Hartogh, P., D. C. Lis, D. Bockelée-Morvan, M. de Val-Borro, N. Biver, M. Küppers, M. Emprechtinger, E. A. Bergin, J. Crovisier, M. Rengel, R. Moreno, S. Szutowicz, and G. A. Blake 2011. Ocean-Like Water in the Jupiter-Family Comet 103P/Hartley 2. Nature 478, 218-220.

Jehin, E., J. Manfroid, D. Hutsemekers, M. Gillon, and P. Magain 2010. Comet 103P/Hartley. Central Bureau Electronic Telegrams 2589.

Knight, M., and D. Schleicher 2009. Comet C/2007 N3 (Lulin). IAU Circ. 9025.

Knight, M., E. Schwieterman, and D. Schleicher 2010. Comet 103P/Hartley. IAU Circ. 9163.

Knight, M. M., T. L. Farnham, D. G. Schleicher, and E. W. Schwieterman 2011. The Increasing Rotation Period of Comet 10P/Tempel 2. AJ 141, 2.

Knight, M. M., and D. G. Schleicher 2011. CN Morphology Studies of Comet 103P/Hartley 2. AJ 141, 183.

Knight, M. M., D. G. Schleicher, T. L. Farnham, E. W. Schwieterman, and S. R. Christensen 2012. Results from Imaging and Photometry of Comet 10P/Tempel 2. AJ, in prep.

Lara, L. M., Z.-Y. Lin, and K. Meech 2011. Comet 103P/Hartley 2 at perihelion: gas and dust activity. $A \mathscr{E} A \mathbf{5 3 2}$, A87.

Lederer, S. M., H. Campins, and D. J. Osip 2009. Chemical and Physical Properties of Gas Jets in Comets. II. Modeling OH, CN and $\mathrm{C}_{2}$ Jets in Comet C/1995 O1 (Hale Bopp) One Month After Perihelion. Icarus 199, 484-504. 
Lederer, S. M., H. Campins, D. J. Osip, and D. G. Schleicher 1997. Gaseous Jets in Comet Hale-Bopp (1995 O1). Earth Moon and Planets 78, 131-136.

Lisse, C. M., D. J. Christian, S. Wolk, D. Bodewits, K. Dennerl, M. Combi, S. Lepri, T. Zurbuchen, and M. Knight 2012. Chandra ACIS-S X-ray Imaging Spectroscopy of Comet 103P/Hartley 2. Icarus, in prep.

Lisse, C. M., Y. R. Fernandez, W. T. Reach, J. M. Bauer, M. F. A'Hearn, T. L. Farnham, O. Groussin, M. J. Belton, K. J. Meech, and C. D. Snodgrass 2009. Spitzer Space Telescope Observations of the Nucleus of Comet 103P/Hartley 2. PASP 121, 968-975.

Meech, K. J., M. F. A'Hearn, J. A. Adams, P. Bacci, J. Bai, L. Barrera, M. Battelino, J. M. Bauer, E. Becklin, B. Bhatt, N. Biver, D. Bockelée-Morvan, D. Bodewits, H. Böhnhardt, J. Boissier, B. P. Bonev, W. Borghini, J. R. Brucato, E. Bryssinck, M. W. Buie, H. Canovas, D. Castellano, S. B. Charnley, W. P. Chen, P. Chiang, Y.-J. Choi, D. J. Christian, Y.-L. Chuang, A. L. Cochran, P. Colom, M. R. Combi, I. M. Coulson, J. Crovisier, N. Dello Russo, K. Dennerl, K. DeWahl, M. A. DiSanti, M. Facchini, T. L. Farnham, Y. Fernández, H. G. Florén, U. Frisk, T. Fujiyoshi, R. Furusho, T. Fuse, G. Galli, D. A. García-Hernández, A. Gersch, Z. Getu, E. L. Gibb, M. Gillon, E. Guido, R. A. Guillermo, E. Hadamcik, O. Hainaut, H. B. Hammel, D. E. Harker, J. K. Harmon, W. M. Harris, P. Hartogh, M. Hashimoto, B. Häusler, T. Herter, A. Hjalmarson, S. T. Holland, M. Honda, S. Hosseini, E. S. Howell, N. Howes, H. H. Hsieh, H.-Y. Hsiao, D. Hutsemékers, S. M. Immler, W. M. Jackson, S. V. Jeffers, E. Jehin, T. J. Jones, M. d. J. Ovelar, H. M. Kaluna, T. Karlsson, H. Kawakita, J. V. Keane, L. D. Keller, M. S. Kelley, D. Kinoshita, N. N. Kiselev, J. Kleyna, M. M. Knight, H. Kobayashi, H. A. Kobulnicky, L. Kolokolova, M. Kreiny, Y.-J. Kuan, M. Küppers, J. M. Lacruz, W. B. Landsman, L. M. Lara, A. Lecacheux, A. C. Levasseur-Regourd, B. Li, J. Licandro, R. Ligustri, Z.-Y. Lin, M. Lippi, D. C. Lis, C. M. Lisse, A. J. Lovell, S. C. Lowry, H. Lu, S. Lundin, K. Magee-Sauer, P. Magain, J. Manfroid, E. Mazzotta Epifani, A. McKay, M. D. Melita, H. Mikuz, S. N. Milam, G. Milani, M. Min, R. Moreno, B. E. A. Mueller, M. J. Mumma, M. Nicolini, M. C. Nolan, H. L. Nordh, P. B. Nowajewski, Odin Team, T. Ootsubo, L. Paganini, C. Perrella, J. Pittichová, E. Prosperi, Y. L. Radeva, W. T. Reach, A. J. Remijan, M. Rengel, T. E. Riesen, M. Rodenhuis, D. P. Rodríguez, R. W. Russell, D. K. Sahu, N. H. Samarasinha, A. Sánchez Caso, A. Sandqvist, G. Sarid, M. Sato, D. G. Schleicher, E. W. Schwieterman, A. K. Sen, D. Shenoy, J.-C. Shi, Y. Shinnaka, J. Skvarc, C. Snodgrass, M. L. Sitko, S. Sonnett, S. Sosseini, G. Sostero, S. Sugita, B. M. Swinyard, S. Szutowicz, N. Takato, P. Tanga, P. A. Taylor, G.-P. Tozzi, R. Trabatti, J. M. Trigo-Rodríguez, C. Tubiana, M. de Val-Borro, W. Vacca, B. Vandenbussche, 
J. Vaubaillion, F. P. Velichko, S. F. Velichko, R. J. Vervack, Jr., M. J. Vidal-Nunez, G. L. Villanueva, C. Vinante, J.-B. Vincent, M. Wang, L. H. Wasserman, J. Watanabe, H. A. Weaver, P. R. Weissman, S. Wolk, D. H. Wooden, C. E. Woodward, M. Yamaguchi, T. Yamashita, P. A. Yanamandra-Fischer, B. Yang, J.-S. Yao, D. K. Yeomans, T. Zenn, H. Zhao, and J. E. Ziffer 2011. EPOXI: Comet 103P/Hartley 2 Observations from a Worldwide Campaign. ApJ 734, L1.

Mueller, B. E. A., N. H. Samarasinha, T. L. Farnham, and M. F. A'Hearn 2012. The Sunward Continuum Features of Comet 103P/Hartley 2. AAS Meeting 220, Abstract \#120.03.

Mumma, M. J., B. P. Bonev, G. L. Villanueva, L. Paganini, M. A. DiSanti, E. L. Gibb, J. V. Keane, K. J. Meech, G. A. Blake, R. S. Ellis, M. Lippi, H. Boehnhardt, and K. Magee-Sauer 2011. Temporal and Spatial Aspects of Gas Release During the 2010 Apparition of Comet 103P/Hartley 2. ApJ 734, L7.

Samarasinha, N. H., B. E. A. Mueller, M. F. A'Hearn, T. L. Farnham, and A. Gersch 2011. Rotation of Comet 103P/Hartley 2 from Structures in the Coma. ApJ 734, L3.

Schleicher, D. G. 2006. Compositional and Physical Results for Rosetta's New Target Comet 67P/Churyumov Gerasimenko from Narrowband Photometry and Imaging. Icarus 181, 442-457.

Schleicher, D. G., and A. N. Bair 2011. The Composition of the Interior of Comet 73P/Schwassmann-Wachmann 3: Results from Narrowband Photometry of Multiple Components. AJ 141, 177.

Schleicher, D. G., K. L. Barnes, and N. F. Baugh 2006. Photometry and Imaging Results for Comet 9P/Tempel 1 and Deep Impact: Gas Production Rates, Postimpact Light Curves, and Ejecta Plume Morphology. AJ 131, 1130-1137.

Schleicher, D. G., R. L. Millis, and P. V. Birch 1998. Narrowband Photometry of Comet P/Halley: Variation with Heliocentric Distance, Season, and Solar Phase Angle. Icarus 132, 397-417.

Schleicher, D. G., and L. M. Woodney 2003. Analyses of Dust Coma Morphology of Comet Hyakutake (1996 B2) Near Perigee: Outburst Behavior, Jet Motion, Source Region Locations, and Nucleus Pole Orientation. Icarus 162, 190-213.

Schleicher, D. G., L. M. Woodney, and R. L. Millis 2003. Comet 19P/Borrelly at Multiple Apparitions: Seasonal Variations in Gas Production and Dust Morphology. Icarus 162, 415-442. 
Schulz, R., and M. F. A'Hearn 1995. Shells in the C2 Coma of Comet P/Halley. Icarus 115, 191-198.

Waniak, W., G. Borisov, M. Drahus, T. Bonev, K. Czart, and M. Küppers 2009. Rotation of the Nucleus, Gas Kinematics and Emission Pattern of Comet 8P/Tuttle: Preliminary Results from Optical Imaging of the CN Coma. Earth Moon and Planets 105, 327342.

Waniak, W., Borisov, G., Drahus, M., \& Bonev, T. 2012, A\&A, accepted.

Weaver, H. A., P. D. Feldman, M. F. A'Hearn, N. Dello Russo, and S. A. Stern 2011. The Carbon Monoxide Abundance in Comet 103P/Hartley 2 During the EPOXI Flyby. ApJ 734, L5.

Weaver, H. A., P. D. Feldman, J. B. McPhate, M. F. A'Hearn, C. Arpigny, and T. E. Smith 1994. Detection of CO Cameron Band Emission in Comet P/Hartley 2 (1991 XV) with the Hubble Space Telescope. ApJ 422, 374-380.

Woodney, L. M., M. F. A'Hearn, D. G. Schleicher, T. L. Farnham, J. P. McMullin, M. C. H. Wright, J. M. Veal, L. E. Snyder, I. de Pater, J. R. Forster, P. Palmer, Y. Kuan, W. R. Williams, C. C. Cheung, and B. R. Smith 2002. Morphology of HCN and CN in Comet Hale-Bopp (1995 O1). Icarus 157, 193-204.

This preprint was prepared with the AAS LATEX macros v5.2. 
Table 1. Summary of Hartley 2 imaging observations and geometric parameters. ${ }^{\text {a }}$

\begin{tabular}{|c|c|c|c|c|c|c|c|c|c|}
\hline $\begin{array}{c}\text { UT } \\
\text { Date }\end{array}$ & $\begin{array}{c}\text { UT } \\
\text { Range }\end{array}$ & $\begin{array}{l}\text { Telescope } \\
\text { Diam. (m) }\end{array}$ & $\begin{array}{c}\Delta \mathrm{T} \\
\text { (days) }\end{array}$ & $\begin{array}{l}r_{\mathrm{H}} \\
(\mathrm{AU})\end{array}$ & $\underset{(\mathrm{AU})}{\Delta}$ & $\begin{array}{c}\theta^{\mathrm{b}} \\
\left({ }^{\circ}\right)\end{array}$ & $\underset{\left({ }^{\circ}\right)}{\mathrm{PA} \operatorname{Sun}^{\mathrm{C}}}$ & Filters & Conditions \\
\hline 2010 Jul 19 & $10: 00-10: 22$ & 1.1 & -100.8 & 1.68 & 0.86 & 29 & 44 & $\mathrm{R}, \mathrm{CN}$ & Thin cirrus \\
\hline 2010 Aug 13 & $03: 28-11: 48$ & $\begin{array}{l}1.1 \\
1.1\end{array}$ & -75.9 & 1.46 & 0.57 & 30 & 22 & $\mathrm{R}, \mathrm{CN}, \mathrm{BC}, \mathrm{C} 3, \mathrm{C} 2, \mathrm{GC}$ & Photometric \\
\hline 2010 Aug 14 & $03: 03-11: 48$ & 1.1 & -74.9 & 1.45 & 0.56 & 30 & 21 & $\mathrm{R}, \mathrm{CN}, \mathrm{BC}, \mathrm{C} 3, \mathrm{C} 2, \mathrm{GC}$ & Photometric \\
\hline 2010 Aug 15 & $03: 15-12: 10$ & 1.1 & -73.9 & 1.45 & 0.55 & 30 & 20 & $\mathrm{R}, \mathrm{CN}, \mathrm{BC}$ & Clouds \\
\hline 2010 Aug 16 & $07: 40-11: 54$ & 1.1 & -72.8 & 1.44 & 0.54 & 31 & 19 & R,CN,BC & Clouds \\
\hline 2010 Aug 17 & $07: 48-11: 01$ & 1.1 & -71.9 & 1.43 & 0.53 & 31 & 18 & R,CN,BC & Clouds \\
\hline 2010 Sep 9 & $02: 53-12: 16$ & 1.1 & -48.9 & 1.25 & 0.33 & 37 & 357 & $\mathrm{R}, \mathrm{CN}, \mathrm{BC}, \mathrm{C} 3, \mathrm{C} 2, \mathrm{GC}, \mathrm{OH}$ & Photometric \\
\hline 2010 Sep 10 & $02: 36-12: 09$ & 1.1 & -48.0 & 1.24 & 0.32 & 37 & 357 & $\mathrm{R}, \mathrm{CN}, \mathrm{BC}, \mathrm{C} 3, \mathrm{C} 2, \mathrm{GC}, \mathrm{OH}$ & Photometric \\
\hline 2010 Sep 11 & $02: 33-12: 08$ & 1.1 & -47.0 & 1.24 & 0.31 & 37 & 356 & $\mathrm{R}, \mathrm{CN}, \mathrm{BC}, \mathrm{C} 3, \mathrm{C} 2, \mathrm{GC}, \mathrm{OH}$ & Photometric \\
\hline 2010 Sep 12 & $02: 33-12: 11$ & 1.1 & -46.0 & 1.23 & 0.31 & 38 & 356 & $\mathrm{R}, \mathrm{CN}, \mathrm{BC}$ & Clouds \\
\hline 2010 Sep 13 & $02: 35-12: 11$ & 1.1 & -44.9 & 1.22 & 0.30 & 38 & 355 & $\mathrm{R}, \mathrm{CN}, \mathrm{BC}, \mathrm{C} 3$ & Clouds \\
\hline 2010 Oct 12 & $03: 12-12: 34$ & 0.8 & -15.9 & 1.08 & 0.13 & 49 & 43 & R,CN & Photometric \\
\hline 2010 Oct 13 & $03: 13-12: 43$ & 0.8 & -14.9 & 1.08 & 0.13 & 49 & 48 & $\mathrm{R}, \mathrm{CN}$ & Photometric \\
\hline 2010 Oct 14 & $03: 13-12: 40$ & 0.8 & -13.9 & 1.08 & 0.13 & 50 & 52 & $\mathrm{R}, \mathrm{CN}$ & Photometric \\
\hline 2010 Oct 15 & $03: 06-05: 33$ & 0.8 & -13.1 & 1.07 & 0.13 & 50 & 56 & $\mathrm{R}, \mathrm{CN}$ & Clouds \\
\hline 2010 Oct 16 & $05: 01-12: 21$ & 1.1 & -11.9 & 1.07 & 0.12 & 51 & 61 & $\mathrm{R}, \mathrm{CN}, \mathrm{BC}, \mathrm{C} 3, \mathrm{C} 2, \mathrm{GC}, \mathrm{OH}, \mathrm{UC}, \mathrm{NH}$ & Thin cirrus \\
\hline 2010 Oct 17 & $05: 00-12: 38$ & 1.1 & -10.9 & 1.07 & 0.12 & 51 & 65 & R,CN,BC & Clouds \\
\hline 2010 Oct 19 & $10: 56-12: 24$ & 1.1 & -8.8 & 1.07 & 0.12 & 52 & 73 & $\mathrm{R}, \mathrm{CN}, \mathrm{BC}$ & Clouds \\
\hline 2010 Oct 31 & $07: 10-12: 36$ & 0.8 & +3.2 & 1.06 & 0.14 & 58 & 99 & $\mathrm{R}, \mathrm{CN}$ & Thin cirrus \\
\hline 2010 Nov 1 & $07: 15-12: 45$ & 0.8 & +4.2 & 1.06 & 0.14 & 59 & 100 & $\mathrm{R}, \mathrm{CN}$ & Thin cirrus \\
\hline 2010 Nov 2 & $06: 45-12: 54$ & 1.1 & +5.2 & 1.06 & 0.15 & 59 & 102 & $\mathrm{R}, \mathrm{CN}, \mathrm{BC}, \mathrm{C} 3, \mathrm{C} 2, \mathrm{GC}, \mathrm{OH}, \mathrm{UC}, \mathrm{NH}, \mathrm{RC}$ & Photometric \\
\hline 2010 Nov 2 & $07: 27-10: 32$ & 0.8 & +5.1 & 1.06 & 0.15 & 59 & 102 & $\mathrm{R}, \mathrm{CN}$ & Photometric \\
\hline 2010 Nov 3 & $06: 41-13: 01$ & 1.1 & +6.2 & 1.06 & 0.15 & 59 & 103 & $\mathrm{R}, \mathrm{CN}, \mathrm{BC}, \mathrm{C} 3, \mathrm{C} 2, \mathrm{GC}, \mathrm{OH}, \mathrm{UC}, \mathrm{NH}, \mathrm{RC}$ & Photometric \\
\hline 2010 Nov 4 & $06: 39-13: 07$ & 1.1 & +7.2 & 1.06 & 0.16 & 59 & 104 & $\mathrm{R}, \mathrm{CN}, \mathrm{BC}, \mathrm{C} 3, \mathrm{C} 2, \mathrm{GC}, \mathrm{OH}, \mathrm{UC}, \mathrm{H} 20+, \mathrm{RC}$ & Thin cirrus \\
\hline 2010 Nov 5 & $07: 44-10: 45$ & 0.8 & +8.1 & 1.06 & 0.16 & 59 & 105 & $\mathrm{R}, \mathrm{CN}$ & Photometric \\
\hline 2010 Nov 6 & $07: 41-10: 58$ & 0.8 & +9.1 & 1.07 & .16 & 59 & 106 & $\mathrm{R}, \mathrm{CN}$ & Thin cirrus \\
\hline 2010 Nov 7 & $06: 48-13: 09$ & 1.1 & +10.2 & 1.07 & .17 & 59 & 107 & $\mathrm{R}, \mathrm{CN}, \mathrm{BC}, \mathrm{C} 3, \mathrm{C} 2, \mathrm{GC}, \mathrm{OH}, \mathrm{UC}, \mathrm{NH}, \mathrm{RC}$ & Photometric \\
\hline 2010 Nov 10 & $07: 59-13: 11$ & 0.8 & +13.2 & 1.07 & .18 & 58 & 109 & $\mathrm{R}, \mathrm{CN}$ & Clouds \\
\hline 2010 Nov 12 & $07: 56-12: 30$ & 0.8 & +15.2 & 1.08 & 0.19 & 58 & 111 & $\mathrm{R}, \mathrm{CN}$ & Clouds \\
\hline 2010 Nov 13 & $08: 09-13: 10$ & 0.8 & +16.2 & 1.08 & 0.20 & 57 & 112 & $\mathrm{R}, \mathrm{CN}$ & Clouds \\
\hline 2010 Nov 16 & $08: 08-13: 08$ & 0.8 & +19.2 & 1.09 & 0.21 & 56 & 114 & $\mathrm{R}, \mathrm{CN}$ & Photometric \\
\hline 2010 Nov 26 & $07: 45-12: 43$ & 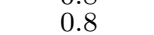 & $\begin{array}{r}1 \\
+29.2 \\
\end{array}$ & 1.13 & 0.26 & 51 & 123 & $\mathrm{R}, \mathrm{CN}$ & Photometric \\
\hline 2010 Nov 27 & $07: 48-12: 46$ & 0 . & +30.2 & 1.14 & 0.27 & 50 & 124 & R,CN & Photometric \\
\hline 2010 Dec 9 & $07: 02-13: 19$ & 1. & $\begin{array}{r}+42.2 \\
+\end{array}$ & 1.21 & 0.33 & 42 & 136 & $\mathrm{R}, \mathrm{CN}, \mathrm{BC}$ & Thin cirrus \\
\hline 2010 Dec 10 & $06: 48-08: 50$ & 1. & $\begin{array}{r}13.1 \\
+43.1\end{array}$ & 1.21 & 0.34 & 41 & 137 & R,CN,BC & Thin cirrus \\
\hline 2010 Dec 15 & $08: 57-09: 13$ & 1. & $\begin{array}{r}18.1 \\
+48.1\end{array}$ & 1.25 & 0.36 & 38 & 143 & $\mathrm{CN}$ & Clouds \\
\hline 2011 Jan 7 & $04: 02-10: 51$ & 1.1 & $\begin{array}{r}1 \\
+71.1\end{array}$ & 1.42 & 0.51 & 25 & 179 & R,CN,BC & Clouds \\
\hline 2011 Jan 8 & $07: 12-07: 57$ & 1.1 & $\begin{array}{r}1.1 \\
+72.1\end{array}$ & 1.43 & 0.52 & 25 & 181 & R,CN & Clouds \\
\hline 2011 Jan 9 & $07: 09-08: 47$ & 1.1 & +73.1 & 1.44 & 0.53 & 25 & 183 & $\mathrm{R}, \mathrm{CN}$ & Clouds \\
\hline 2011 Jan 11 & $04: 01-10: 50$ & 1.1 & $\begin{array}{r}75.1 \\
+75.1\end{array}$ & 1.45 & 0.54 & 24 & 187 & $\mathrm{R}, \mathrm{CN}, \mathrm{BC}$ & Thin cirrus \\
\hline
\end{tabular}

a All parameters are given for the midpoint of each night's observations, and all images were obtained at Lowell Observatory.

${ }^{\mathrm{b}}$ Phase angle.

${ }^{\mathrm{c}}$ Position angle of the Sun, measured counterclockwise from north through east. 
Table 2. Photometry observing circumstances and fluorescence efficiencies for Comet 103P/Hartley 2. ${ }^{\mathrm{a}}$

\begin{tabular}{|c|c|c|c|c|c|c|c|c|c|c|c|}
\hline \multicolumn{3}{|c|}{ UT Date } & \multirow{2}{*}{$\begin{array}{l}\Delta \mathrm{T}^{\mathrm{b}} \\
\text { (day) }\end{array}$} & \multirow{2}{*}{$\begin{array}{c}r_{\mathrm{H}} \\
(\mathrm{AU})\end{array}$} & \multirow{2}{*}{$\begin{array}{c}\Delta \\
(\mathrm{AU})\end{array}$} & \multirow{2}{*}{$\begin{array}{c}\text { Phase } \\
\text { Angle }\left(^{\circ}\right)\end{array}$} & \multirow{2}{*}{$\begin{array}{l}\text { Phase Adj. } \\
\log A\left(0^{\circ}\right) f \rho^{\mathrm{c}}\end{array}$} & \multirow{2}{*}{$\begin{array}{c}\dot{r}_{\mathrm{H}} \\
\left(\mathrm{km} \mathrm{s}^{-1}\right)\end{array}$} & \multicolumn{3}{|c|}{$\bar{c}_{\log L / N\left(\operatorname{erg~s}^{-1} \text { molecule }^{-1}\right)}$} \\
\hline & & & & & & & & & $\mathrm{OH}$ & $\mathrm{NH}$ & $\mathrm{CN}$ \\
\hline $\begin{array}{l}1991 \\
1991\end{array}$ & $\begin{array}{l}\text { Oct } \\
\text { Oct }\end{array}$ & $\begin{array}{r}8.49 \\
11.44\end{array}$ & $\begin{array}{l}+27.2 \\
+30.2\end{array}$ & $\begin{array}{l}1.033 \\
1.050\end{array}$ & $\begin{array}{l}1.046 \\
1.063\end{array}$ & $\begin{array}{l}57.5 \\
56.4\end{array}$ & $\begin{array}{l}+0.47 \\
+0.47\end{array}$ & $\begin{array}{r}+9.7 \\
+10.5\end{array}$ & $\begin{array}{l}-14.544 \\
-14.507\end{array}$ & $\begin{array}{l}-13.096 \\
-13.121\end{array}$ & $\begin{array}{l}-12.351 \\
-12.370\end{array}$ \\
\hline $\begin{array}{l}1997 \\
1997 \\
1997 \\
1998\end{array}$ & $\begin{array}{l}\text { Nov } \\
\text { Dec } \\
\text { Dec } \\
\text { Feb }\end{array}$ & $\begin{array}{r}2.13 \\
1.53 \\
4.16 \\
26.23\end{array}$ & $\begin{array}{l}-49.1 \\
-19.7 \\
-17.1 \\
+66.9\end{array}$ & $\begin{array}{l}1.241 \\
1.071 \\
1.062 \\
1.370\end{array}$ & $\begin{array}{l}1.019 \\
0.918 \\
0.908 \\
1.091\end{array}$ & $\begin{array}{l}50.9 \\
58.8 \\
59.4 \\
45.7\end{array}$ & $\begin{array}{l}+0.47 \\
+0.46 \\
+0.46 \\
+0.46\end{array}$ & $\begin{array}{r}-12.7 \\
-6.5 \\
-5.8 \\
+14.4\end{array}$ & $\begin{array}{l}-14.735 \\
-14.839 \\
-14.818 \\
-14.504\end{array}$ & $\begin{array}{l}-13.328 \\
-13.177 \\
-13.172 \\
-13.397\end{array}$ & $\begin{array}{l}-12.648 \\
-12.489 \\
-12.495 \\
-12.642\end{array}$ \\
\hline $\begin{array}{l}2010 \\
2010 \\
2010 \\
2010 \\
2010 \\
2010 \\
2010 \\
2010 \\
2010 \\
2011 \\
2011 \\
2011 \\
2011\end{array}$ & $\begin{array}{l}\text { Jul } \\
\text { Aug } \\
\text { Aug } \\
\text { Sep } \\
\text { Sep } \\
\text { Oct } \\
\text { Oct } \\
\text { Nov } \\
\text { Dec } \\
\text { Jan } \\
\text { Feb } \\
\text { Feb } \\
\text { Feb }\end{array}$ & $\begin{array}{r}12.35 \\
11.30 \\
12.24 \\
7.21 \\
30.16 \\
1.17 \\
31.30 \\
16.38 \\
13.34 \\
5.34 \\
1.17 \\
2.25 \\
23.14\end{array}$ & $\begin{array}{r}-107.9 \\
-78.0 \\
-77.0 \\
-51.1 \\
-28.1 \\
-27.1 \\
+3.0 \\
+19.1 \\
+46.1 \\
+69.1 \\
+95.9 \\
+97.0 \\
+117.9\end{array}$ & $\begin{array}{l}1.742 \\
1.480 \\
1.472 \\
1.266 \\
1.128 \\
1.123 \\
1.060 \\
1.091 \\
1.232 \\
1.405 \\
1.635 \\
1.645 \\
1.831\end{array}$ & $\begin{array}{l}0.958 \\
0.588 \\
0.578 \\
0.344 \\
0.189 \\
0.183 \\
0.140 \\
0.211 \\
0.352 \\
0.497 \\
0.742 \\
0.754 \\
1.025\end{array}$ & $\begin{array}{l}29.0 \\
29.9 \\
30.0 \\
35.8 \\
44.4 \\
44.7 \\
58.2 \\
56.0 \\
39.3 \\
26.0 \\
21.8 \\
21.8 \\
24.2\end{array}$ & $\begin{array}{l}+0.38 \\
+0.38 \\
+0.38 \\
+0.42 \\
+0.46 \\
+0.46 \\
+0.47 \\
+0.47 \\
+0.44 \\
+0.35 \\
+0.31 \\
+0.31 \\
+0.33\end{array}$ & $\begin{array}{r}-15.4 \\
-14.7 \\
-14.7 \\
-12.4 \\
-8.1 \\
-7.9 \\
+1.0 \\
+5.8 \\
+11.6 \\
+14.2 \\
+15.3 \\
+15.3 \\
+15.4\end{array}$ & $\begin{array}{l}-15.215 \\
-15.011 \\
-15.006 \\
-14.745 \\
-14.870 \\
-14.873 \\
-14.857 \\
-14.650 \\
-14.561 \\
-14.532 \\
-14.656 \\
-14.662 \\
-14.757\end{array}$ & $\begin{array}{l}-13.607 \\
-13.471 \\
-13.466 \\
-13.346 \\
-13.220 \\
-13.215 \\
-13.223 \\
-13.135 \\
-13.272 \\
-13.417 \\
-13.558 \\
-13.564 \\
-13.658\end{array}$ & $\begin{array}{l}-12.943 \\
-12.799 \\
-12.793 \\
-12.670 \\
-12.519 \\
-12.516 \\
-12.597 \\
-12.400 \\
-12.527 \\
-12.668 \\
-12.793 \\
-12.799 \\
-12.896\end{array}$ \\
\hline
\end{tabular}

${ }^{a}$ All parameters were taken at the midpoint of each night's observations, and all photometry was obtained at Lowell Observatory except for 1997 Dec 1 which was obtained at Perth Observatory.

b Time from perihelion.

${ }^{\mathrm{c}}$ Adjustment to $0^{\circ}$ phase angle to $A(\theta) f \rho$ values based on assumed phase function (see text). 
Table 3. Photometric fluxes and aperture abundances for Comet 103P/Hartley 2

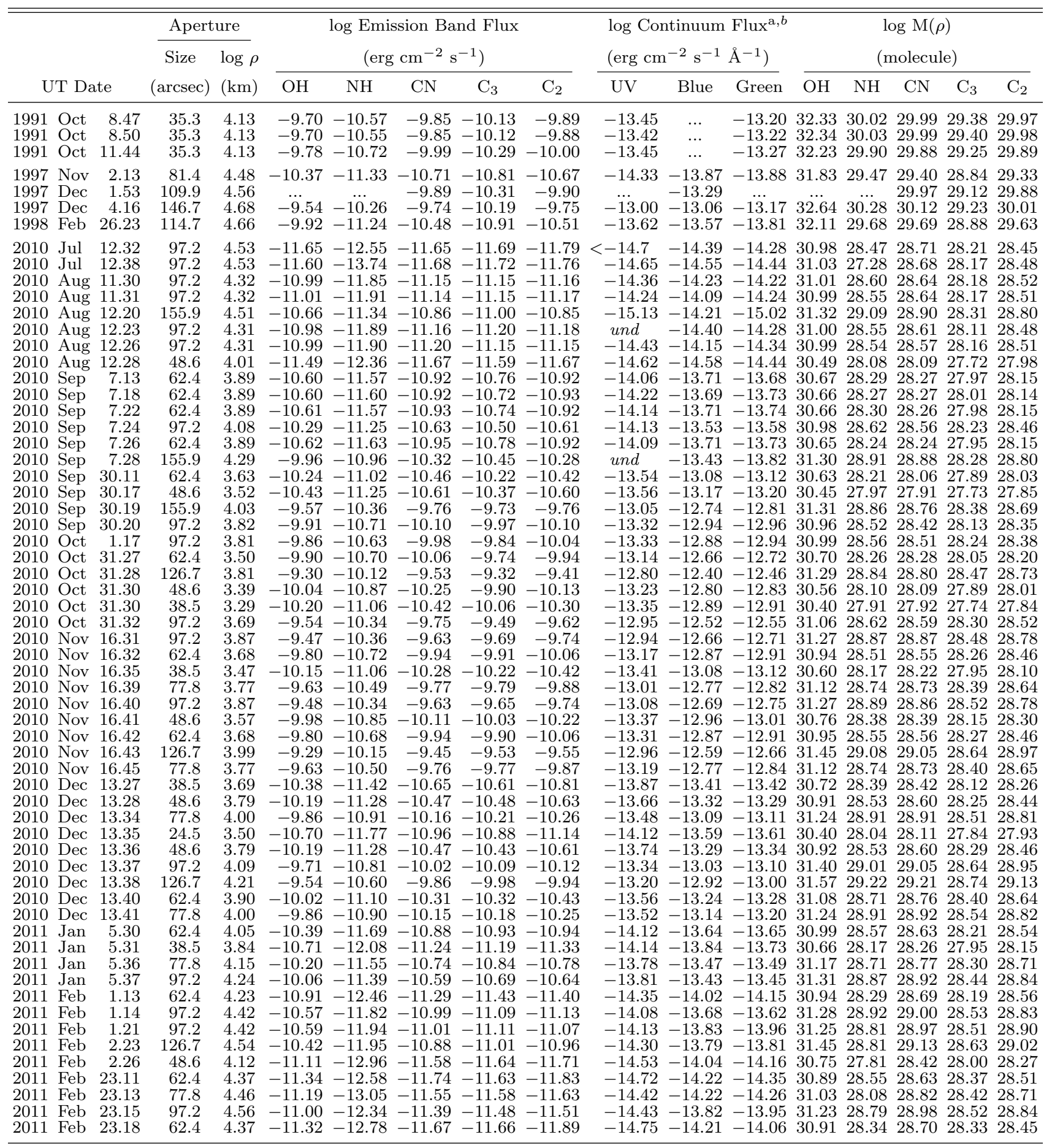




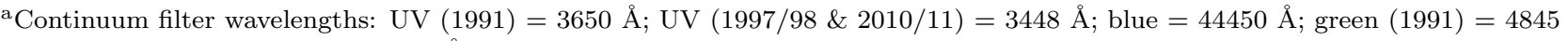
$\AA$; green $(1997 / 98 \& 2010 / 11)=5260 \AA$.

b "und" stands for "undefined" and means the continuum flux was measured but was less than 0. 
Table 4. Photometric production rates for Comet 103P/Hartley 2.

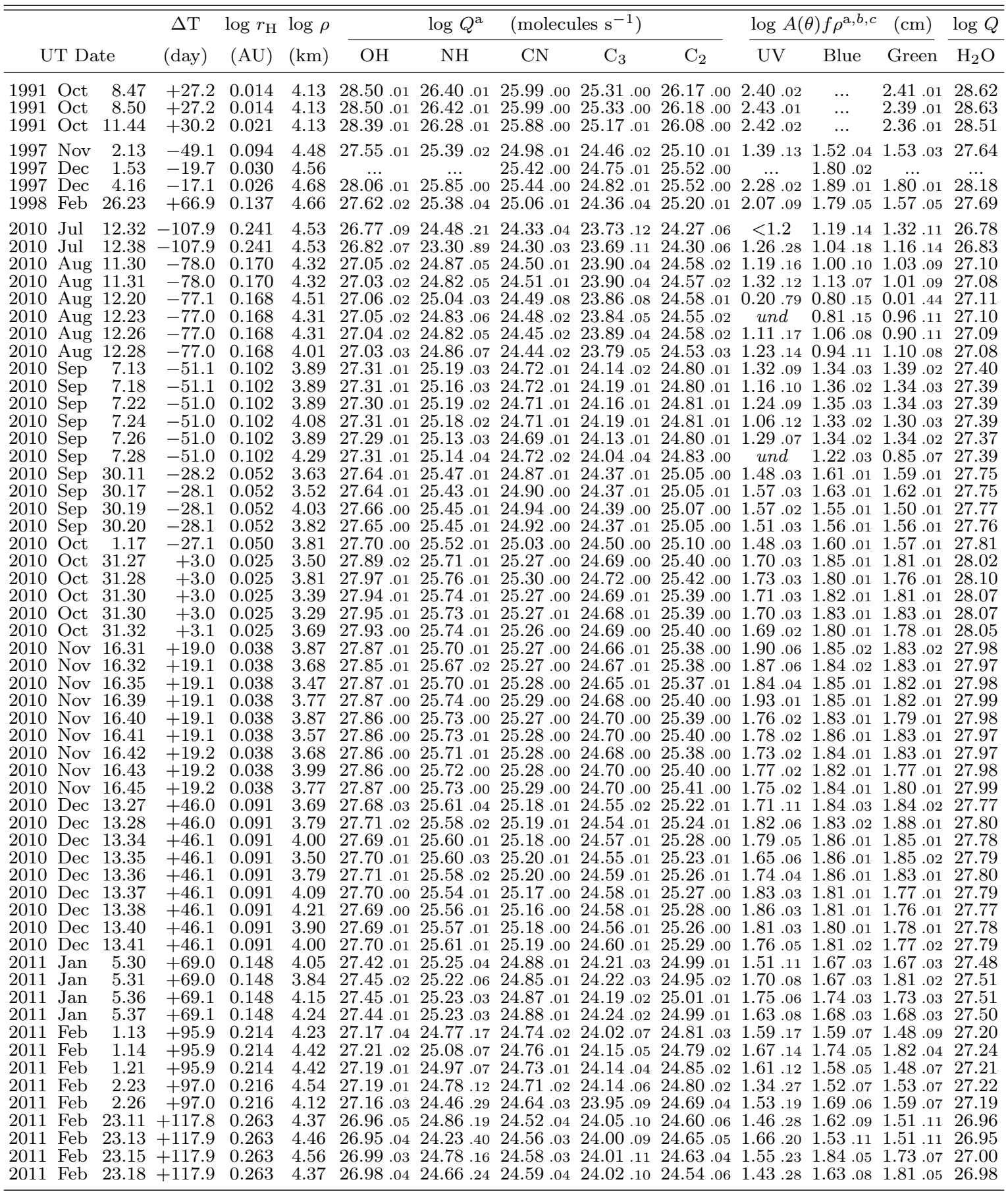


aProduction rates, followed by the upper, i.e. the positive uncertainty. The "+" and "-" uncertainties are equal as percentages, but unequal in log-space; the "-" values can be computed.

${ }^{\mathrm{b}}$ Continuum filter wavelengths: UV $(1991)=3650 \AA$; UV $(1997 / 98 \& 2010 / 11)=3448 \AA$; blue $=4450 \AA$; green $(1991)=4845 \AA$; green $(1997 / 98 \& 2010 / 11)=5260 \AA$.

c "und" stands for "undefined" and means the continuum flux was measured but was less than 0 . 
Table 5. Abundance ratios for 103P/Hartley 2

\begin{tabular}{|c|c|c|c|c|c|}
\hline \multirow[b]{2}{*}{ Species } & \multicolumn{2}{|c|}{$r_{\mathrm{H}}$-dependence } & \multicolumn{3}{|c|}{$\begin{array}{l}\log \text { Production Rate } \\
{\text { Ratios }(\mathrm{X} / \mathrm{OH})^{\mathrm{a}}}\end{array}$} \\
\hline & Pre-Peri & Post-Peri & Mean & $\sigma_{\text {mean }}$ & $\sigma_{\text {data }}$ \\
\hline $\begin{array}{l}\mathrm{OH} \\
\mathrm{NH} \\
\mathrm{CN} \\
\mathrm{C}_{3} \\
\mathrm{C}_{2} \\
\mathrm{UC}^{\mathrm{C}} \\
\mathrm{BC} \\
\mathrm{GC}\end{array}$ & $\begin{array}{l}-4.64 \pm 0.22 \\
-7.08 \pm 0.98 \\
-3.44 \pm 0.18 \\
-3.93 \pm 0.25 \\
-4.10 \pm 0.10 \\
-2.85 \pm 1.26 \\
-3.55 \pm 0.60 \\
-3.35 \pm 1.25\end{array}$ & $\begin{array}{l}-3.99 \pm 0.05 \\
-4.92 \pm 0.27 \\
-3.20 \pm 0.10 \\
-3.20 \pm 0.13 \\
-3.45 \pm 0.09 \\
-1.19 \pm 0.19 \\
-0.99 \pm 0.13 \\
-0.97 \pm 0.17\end{array}$ & $\begin{array}{r}0.00 \\
-2.18 \\
-2.55 \\
-3.15 \\
-2.46 \\
-25.88 \\
-25.81 \\
-25.84\end{array}$ & $\begin{array}{l}+0.01 \\
+0.01 \\
+0.01 \\
+0.01 \\
+0.03 \\
+0.04 \\
+0.04\end{array}$ & $\begin{array}{l}+0.09 \\
+0.07 \\
+0.07 \\
+0.07 \\
+0.23 \\
+0.25 \\
+0.26\end{array}$ \\
\hline $\begin{array}{l}\mathrm{UC}^{\mathrm{b}} \\
\mathrm{BC}^{\mathrm{b}} \\
\mathrm{GC}^{\mathrm{b}}\end{array}$ & $\begin{array}{l}-3.39 \pm 1.28 \\
-4.03 \pm 0.63 \\
-3.84 \pm 1.26\end{array}$ & $\begin{array}{l}-1.91 \pm 0.20 \\
-1.71 \pm 0.15 \\
-1.68 \pm 0.19\end{array}$ & $\begin{array}{l}-25.43 \\
-25.44 \\
-25.48 \\
\end{array}$ & $\begin{array}{l}+0.03 \\
+0.04 \\
+0.03\end{array}$ & $\begin{array}{l}+0.21 \\
+0.22 \\
+0.21\end{array}$ \\
\hline
\end{tabular}

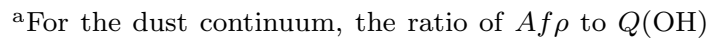
has units of $\mathrm{cm} \mathrm{s} \mathrm{mol}^{-1} . \sigma_{\text {data }}$ is the standard deviation and measures the scatter of the data values around the sample mean, while $\sigma_{\text {mean }}$ is the standard deviation of the sampling distribution of the mean, or the standard deviation divided by the square root of the number of cases. Only upper error bars are given; the lower error bars can be derived if desired.

${ }^{\mathrm{b}}$ Af $\rho$ adjusted to $\theta=0^{\circ}$. 

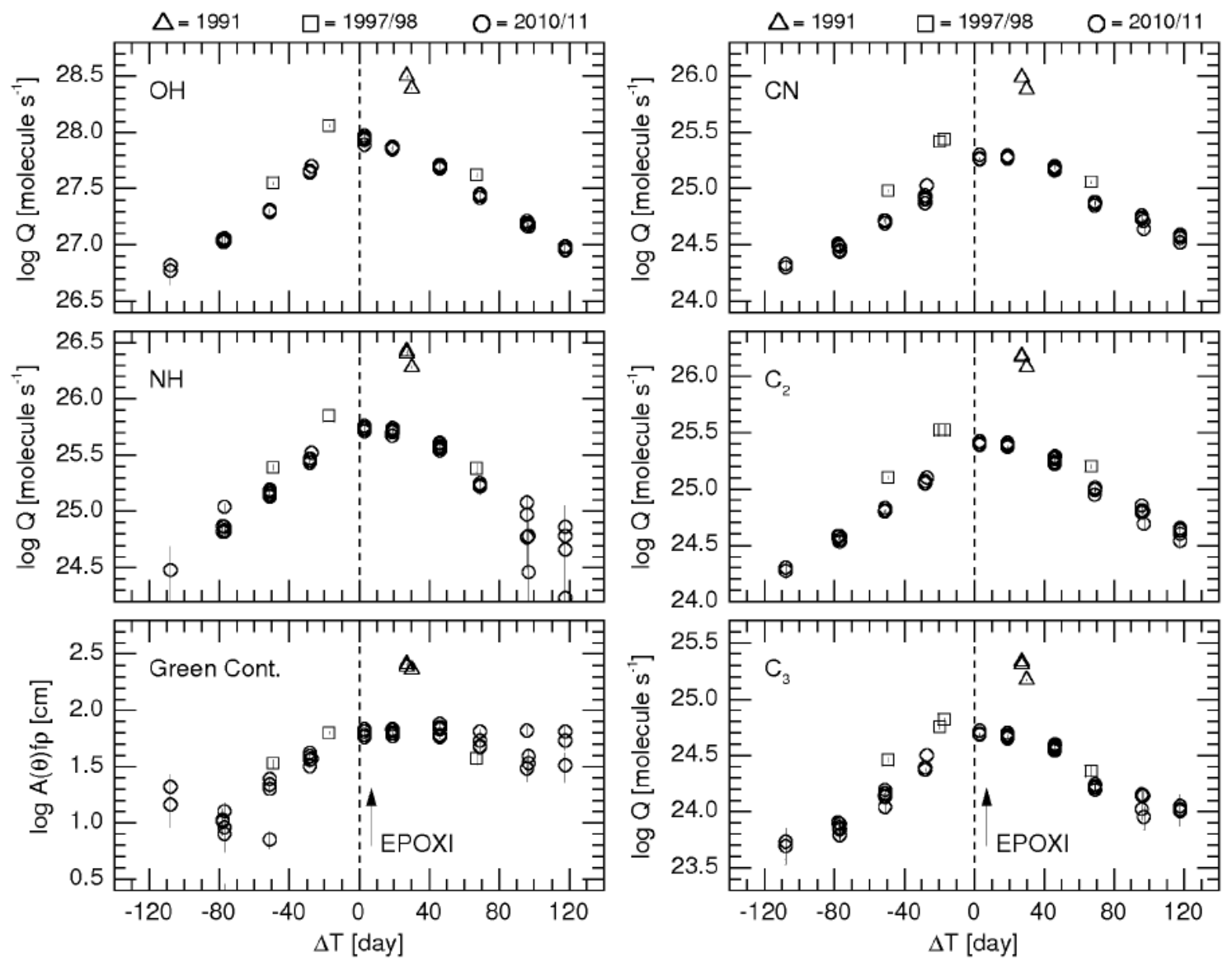

Fig. 1. - Log of the production rates for each observed molecular species and $A(\theta) f \rho$ for the green continuum plotted as a function of time from perihelion. Data points from the 1991 apparition are shown as triangles, those from 1997/98 are given as squares, and the recent 2010/11 data are shown as circles. Error bars are plotted; in cases where they are not visible it is because they are smaller than the symbols. The time of the EPOXI spacecraft flyby is shown with an arrow. Note the large asymmetries around perihelion for all species, and the much shallower dust slope as compared to the gas species following perihelion. Although the perihelion distance increased at each successive apparition, the drop in production rates with apparition is much larger than can be explained by the relatively small drop in solar illumination. 


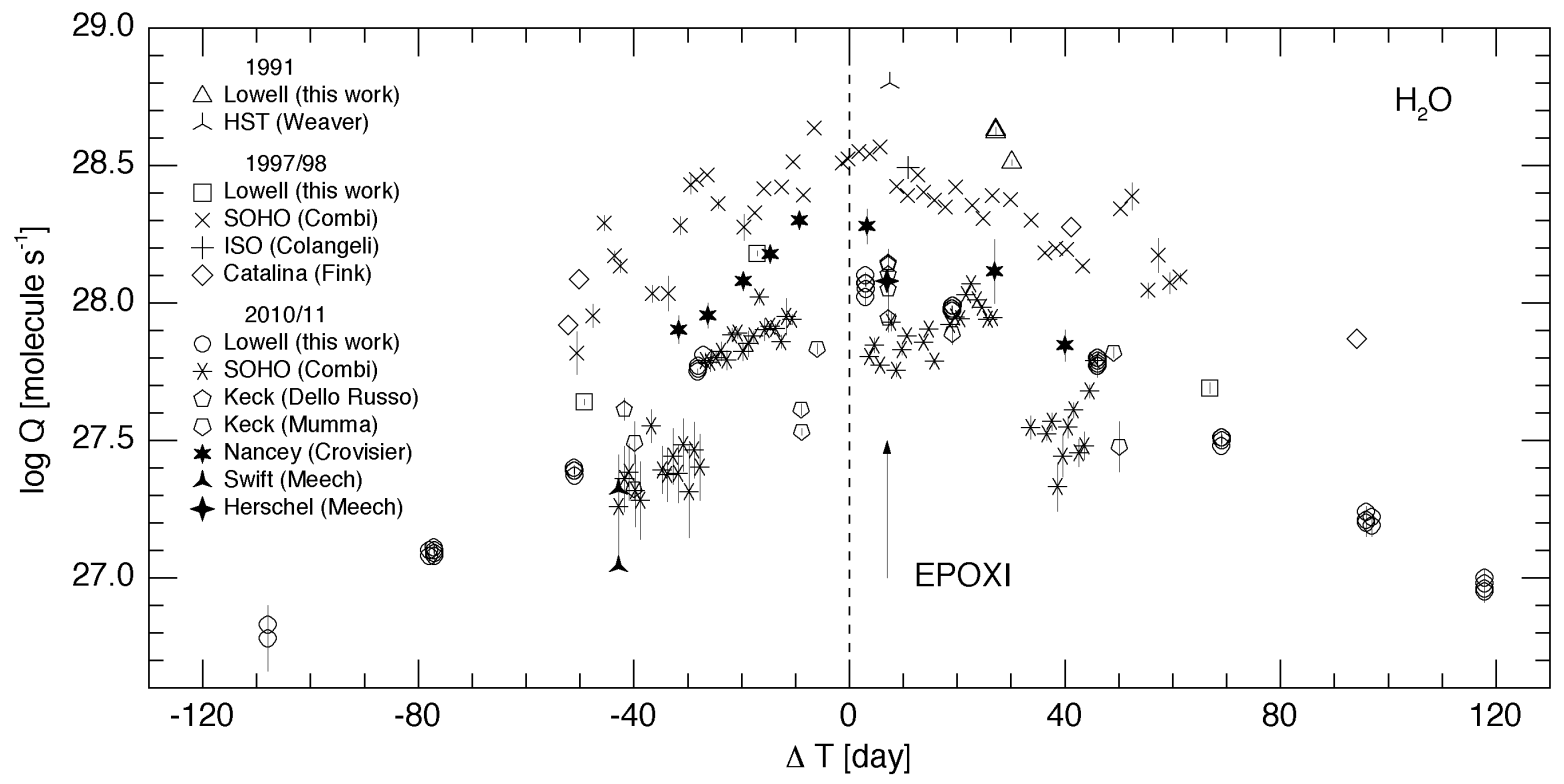

Fig. 2.- Log of the production rates for water plotted as a function of time from perihelion. Our values, based on $\mathrm{OH}$, are shown with the same symbols as Figure 1. Other OH-based results include $H S T / F O S$ data by Weaver et al. (1994), data from the Swift satellite (Bodewits private communication; Meech et al. 2011), and radio $\mathrm{OH}$ measurements from Nancey Crovisier et al. (2012). Forbidden oxygen data were obtained by Fink (2009) at the 1997 apparition, while the granddaughter hydrogen was measured using the Lyman alpha line with SOHO/SWAN (Combi et al. 2011b; Combi et al. 2011a). In the IR, water measurements have become more common, with space-based data from ISO/ISOPHOT (Colangeli et al. 1999) and Herschel (Meech et al. 2011), and ground-based data with Keck/NIRSPEC (Dello Russo et al. 2010, 2011; Mumma et al. 2011). See the key to associate the symbols with these references; only the first author is listed in the key due to space constraints. As discussed in the text, the ensemble of data indicate a larger drop in production rates between 1997/98 and 2010/11 and a larger amount of variability than we inferred from our own data alone. 

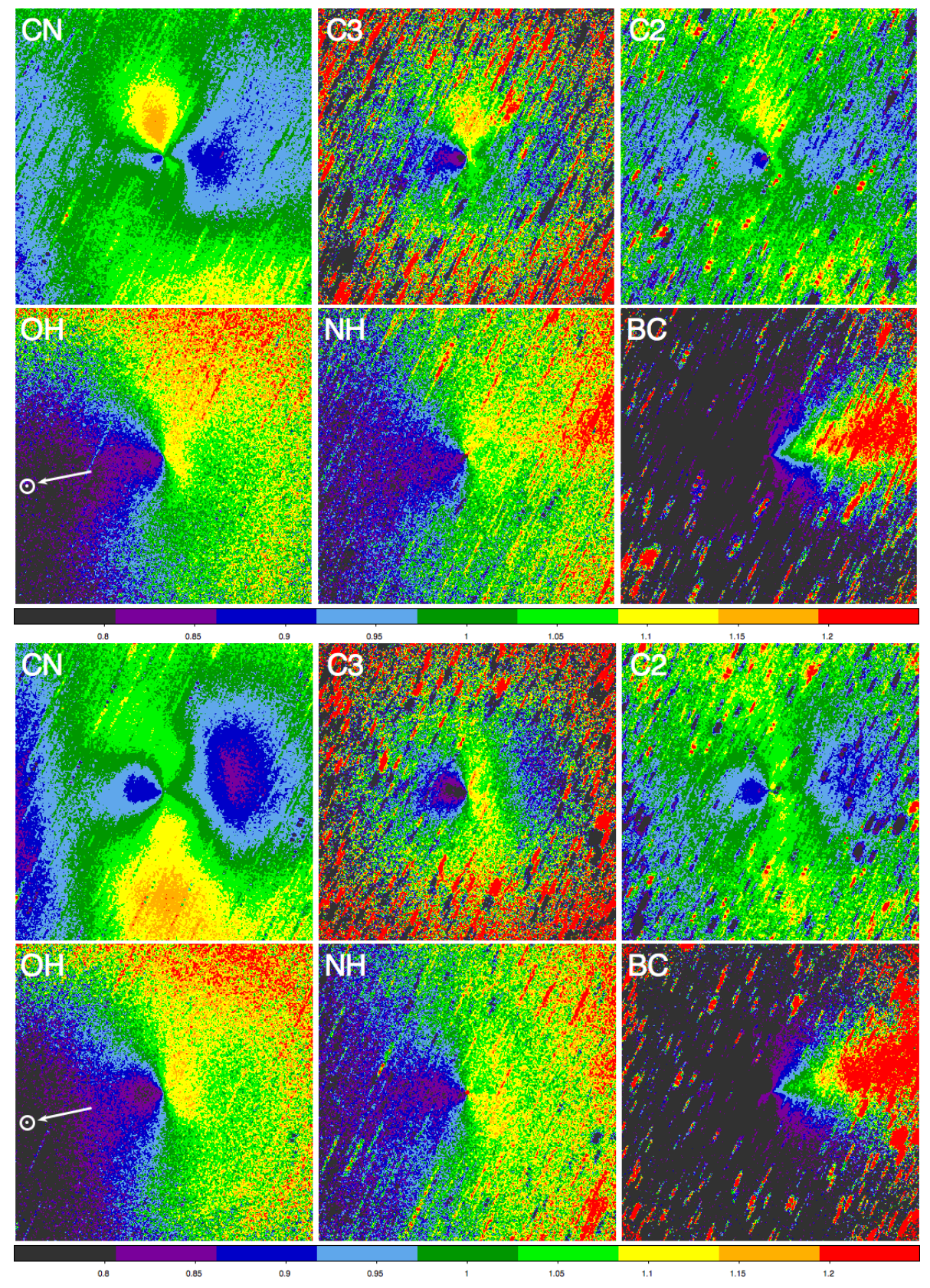

Fig. 3.- Gas and dust coma morphology of 103P/Hartley 2 on 2010 November 2 (top panel) and 2010 November 3 (bottom panel). The bandpass is given in the top left of each image with $\mathrm{BC}$ denoting blue continuum (e.g., dust). Each image is a decontaminated pure gas or dust image centered on the nucleus and enhanced by division of an azimuthal median profile then smoothed with a boxcar smooth. Each image is approximately $64,000 \mathrm{~km}$ across with north up and east to the left. The direction to the Sun is indicated in the $\mathrm{OH}$ frames. The color scale is given at the bottom; the stretch is the same for all gas images, and a different stretch is used for both $\mathrm{BC}$ images. Trailed stars are visible as streaks extending from the northwest to the southeast. Note that some artifacts of the enhancement are visible as ring-like structures 


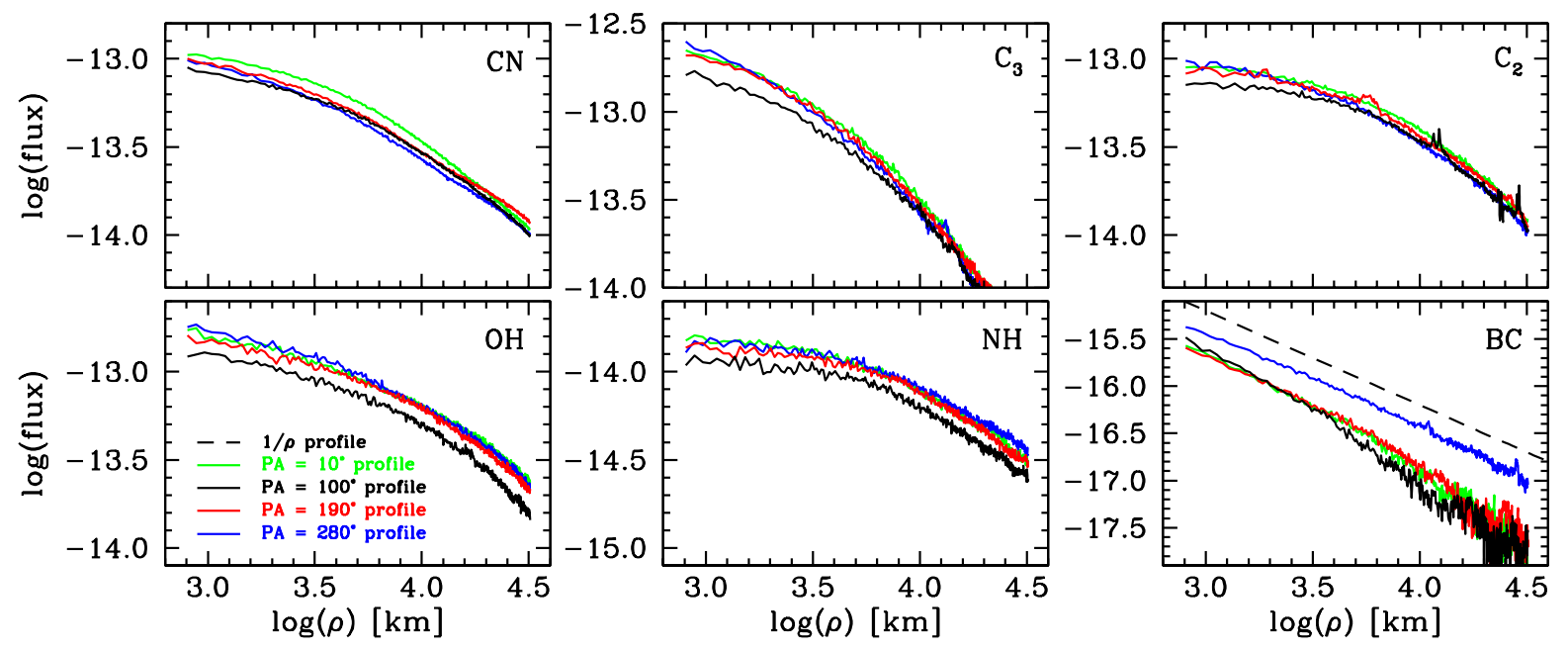

Fig. 4. - Log of the gas and dust flux on 2010 November 2 as a function of the log of the distance from the nucleus in the sunward direction (black), anti-sunward direction (blue), and orthogonal to the sunward line to the north (green) and to the south (red). Each profile is the mean of all points a distance $\rho$ from the nucleus within a $10^{\circ}$ wide wedge centered on a given position angle (PA). The wedges are centered at PAs $10^{\circ}$ (green), $100^{\circ}$ (black), $190^{\circ}$ (red), and $280^{\circ}$ (blue). The sun is at a PA of $102^{\circ}$. The species is given in the top right corner of each plot. The flux has units of erg $\mathrm{cm}^{-2} \mathrm{~s}^{-1}$ for the gas species and $\mathrm{erg} \mathrm{cm} \mathrm{cm}^{-2} \mathrm{~s}^{-1}$ $\AA^{-1}$ for BC. A $1 / \rho$ profile is shown as a dashed line in the BC plot. Note that the slopes of the gas species can be compared directly, as they are plotted with the same $\Delta \log ($ flux $)$, but with different ranges. 

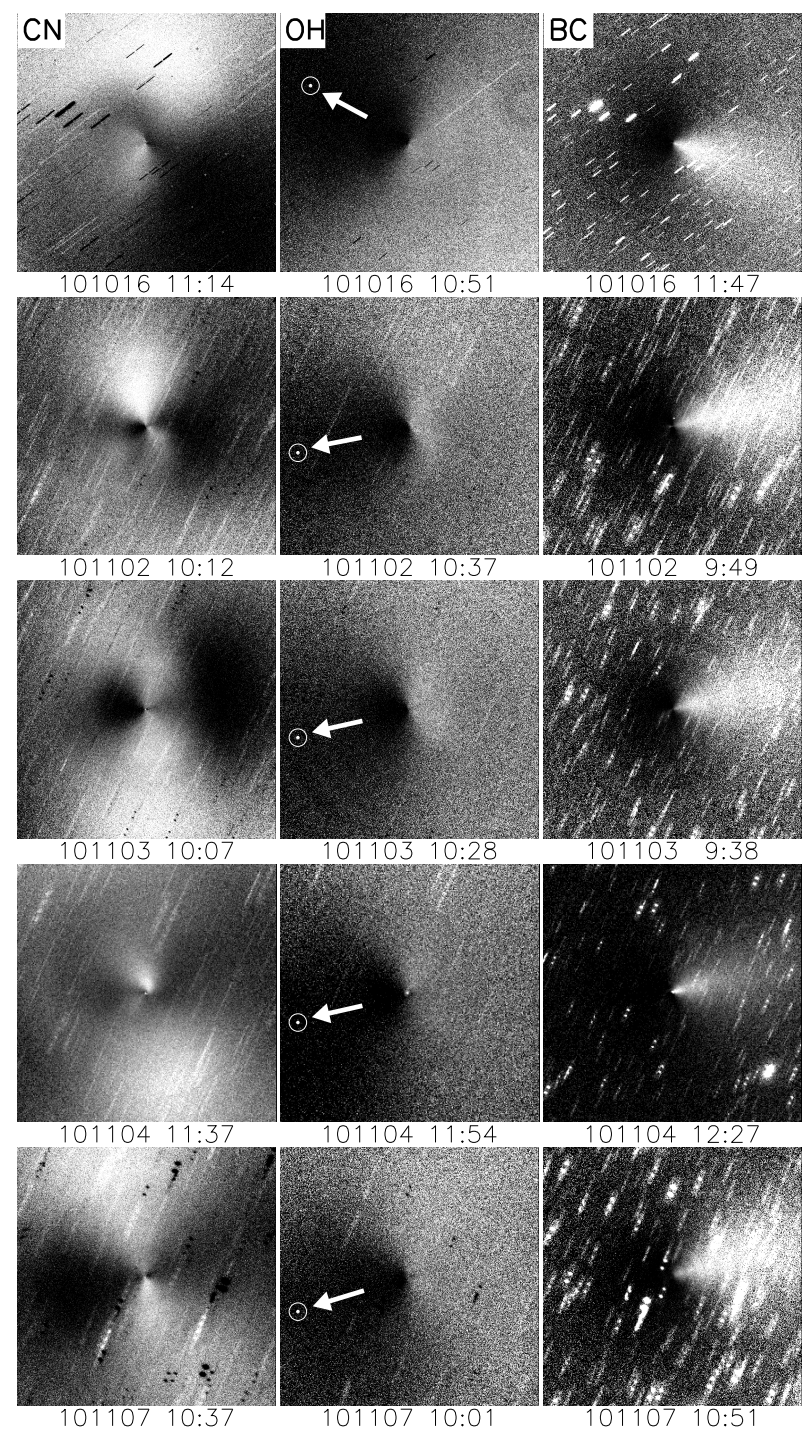

Fig. 5.- CN, OH, and dust continuum of 103P/Hartley 2 in 2010 October and November. The left column is $\mathrm{CN}$, the middle column is $\mathrm{OH}$, and the right column is dust (blue continuum; BC). The date (YYMMDD) and UT time (HH:MM) of the midpoint of each image is below the image. Each image is a decontaminated pure gas or dust image except for 2010 November 4 (the night of the EPOXI flyby) when it was not photometric, and the original image is shown after bias removal and flat-fielding. Each image is centered on the nucleus and enhanced by division of an azimuthal median profile, is approximately $50,000 \mathrm{~km}$ across, and has north up and east to the left. The direction to the Sun is indicated on the OH image for each day. All of the images in a given filter have the same stretch, but the stretch varies from filter to filter. In all cases white is bright and black is dark. Stars appear as trailed streaks, and some images have faint circular artifacts from the enhancement process. The $\mathrm{OH}$ morphology is distinctly different from the CN morphology and is concentrated in the anti-sunward hemisphere, suggesting that a substantial amount of $\mathrm{OH}$ is coming from small, icy grains. 

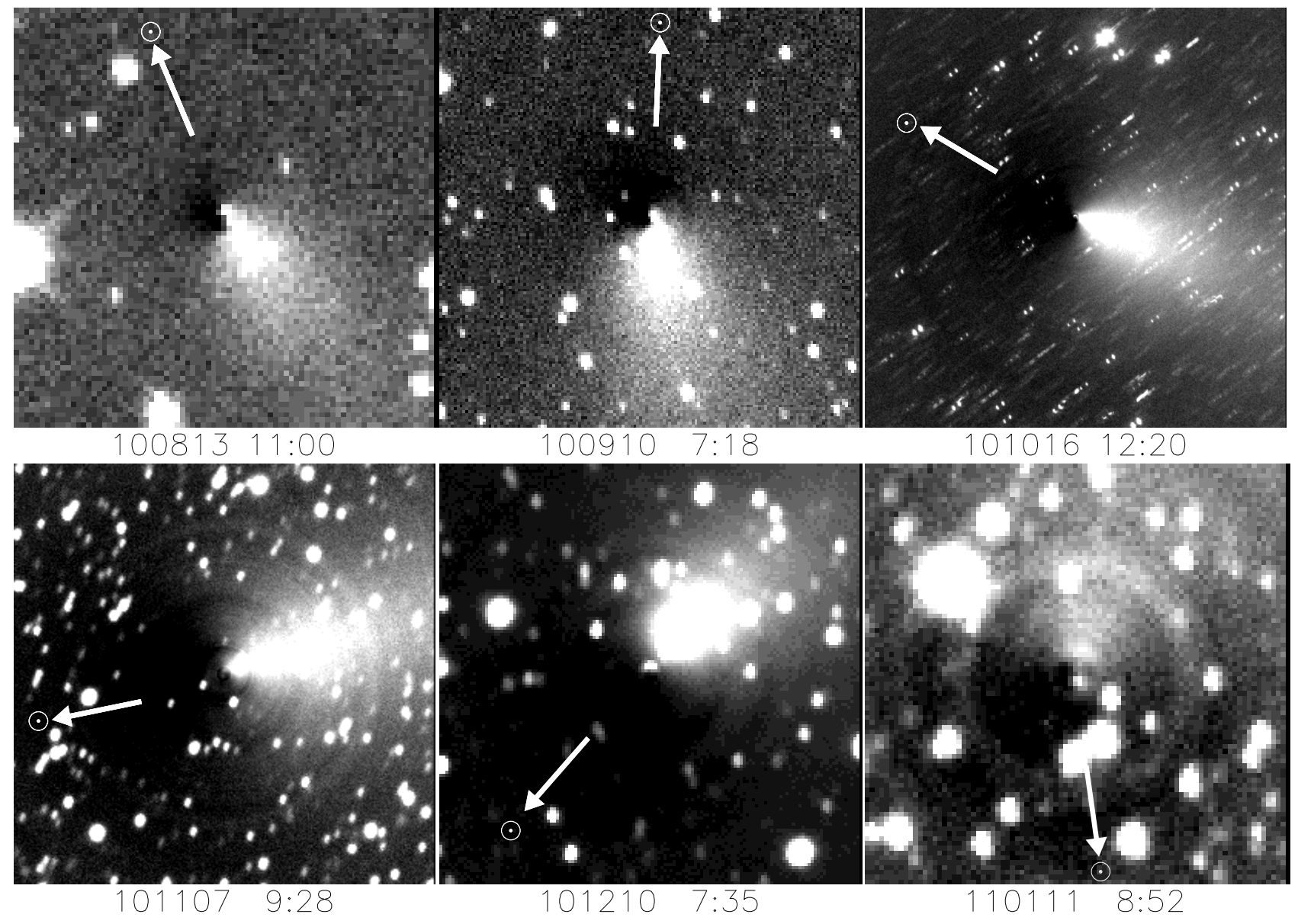

Fig. 6.- Evolution of R-band (dust) morphology of 103P/Hartley 2 from 2010 August until 2011 January. All images are R-band. All other details are as given in Figure 5. Note that some artifacts of the enhancement are visible as ring-like structures. 


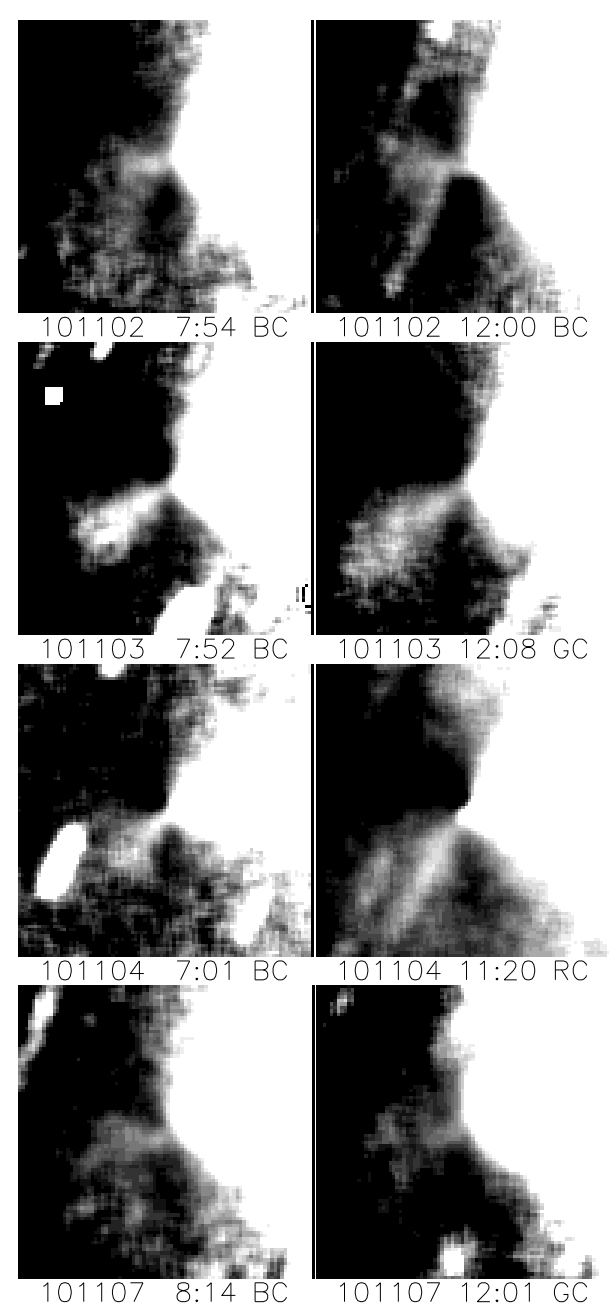

Fig. 7.- Hartley 2 dust jet near the beginning and end of 2010 November 2 (top row), 2010 November 3 (second row), 2010 November 4 (third row), 2010 November 7 (bottom row). Each image is a decontaminated pure dust image except for 2010 November 4 (the night of the EPOXI flyby) when it was not photometric, and the original images are shown after bias removal and flat-fielding. Note that BC images are, by definition, considered to be free of contamination (cf. Farnham et al. 2000). The date (YYMMDD), midpoint UT time (HH:MM), and filter of each image is below the image. Each image is $\sim 8,000 \mathrm{~km}$ across, centered on the nucleus, enhanced by division of an azimuthal median profile, and smoothed with a boxcar smooth. North is up, east is left, and the position angle (PA) of the Sun is near $105^{\circ}$ (exact PAs for each night are given in Table 1). All images have the same stretch. The dust jet can be seen at PAs of $\sim 95^{\circ}$ on November $2, \sim 115^{\circ}$ on November $3, \sim 125^{\circ}$ on November 4 , and $\sim 100^{\circ}$ on November 7 . Diagonal streaks are trailed stars and the bright white area in the west half of each image is the dust tail. 